\title{
Identification of Genetic Markers and Immune Infiltration Characteristics of Alzheimer's Disease Through Weighted Gene Co-expression Network Analysis
}

\section{Qiang Zhang ( $\sim 1625644858 @ q q . c o m)$}

Tianjin Medical University General Hospital, Tianjin Geriatrics Institute

KeFei Duan

Tianjin Medical University General Hospital, Tianjin Geriatrics Institute

YuYang Miao

Tianjin Medical University General Hospital, Tianjin Geriatrics Institute Jin Tan

Tianjin Medical University General Hospital, Tianjin Geriatrics Institute

\section{Research Article}

Keywords: Genetic biomarkers, Weight gene co-expression network analysis(WGCNA), Alzheimer's disease, epigenetic changes, immune infiltration

Posted Date: January 18th, 2022

DOI: https://doi.org/10.21203/rs.3.rs-1113029/v1

License: (c) (i) This work is licensed under a Creative Commons Attribution 4.0 International License. Read Full License 


\section{Identification of genetic markers and immune infiltration}

characteristics of Alzheimer's disease through weighted gene

\section{co-expression network analysis}

Authors: KeFei Duan ${ }^{1}$, Qiang Zhang ${ }^{2}$, YuYang Miao ${ }^{3}$, Jin Tan ${ }^{4}$

Afflications: Department of Geriatrics, Tianjin Medical University General Hospital, Tianjin

Geriatrics Institute, Tianjin, China

Authors:

The First author: KeFei Duan ${ }^{1}$, E-mail: $\underline{970585113 @ q q . c o m}$

The Correspondence author: Qiang Zhang'2, E-mail: 1625644858@qq.com

The Third author: YuYang Miao³, E-mail: 2511261020@qq.com

The Forth author: Jin Tan ${ }^{4}$, E-mail: sispop@qq.com

\section{ABSTRACT:}

Background: Alzheimer disease (AD) is a long-term progressive neurodegeneration disease. Studies have shown that accumulation of epigenetic changes promotes the formation of gene mutations thereby inducing the pathogenesis of Alzheimer disease. Currently, the molecular mechanisms of alzheimer disease are not well defined. This calls for identification of genetic markers of alzheimer disease which can be leveraged to design effective drugs.

Method: In this study, we aimed to determine the modules and hub genes that contribute to the development of alzheimer disease through weighted gene co-expression network analysis (WGCNA). The genes in the module were enriched and analyzed by GO / KEGG, and the protein-protein interaction ( PPI ) network was constructed. In addition, WGCNA topology analysis was carried out to construct a gene network, from which five hub genes were identified. Logistic regression analysis and Receiver operating characteristics (ROC) was performed to explore the clinical value of genes in diagnosis of Alzheimer. The genes in the core module are intersected with the hub genes, and four intersecting genes were selected, which were ATP2A2, ATP6V1D, CAP2 and SYNJ1. The four genes were enriched by GSEA. Finally, immune infiltration analysis was performed. 
Results: The GO/KEGG analysis shows that the genes in the core module play a role in the differentiation and growth of nerve cells and neurotransmitter transmission.Gene set enrichment analysis (GSEA) about core genes demonstrated that the four genes were mainly enriched in immune infection pathways such as cholera infection and Helicobacter pylori infection and other metabolic pathways. In addition, immune infiltration characteristics were investigated and found that $\mathrm{T}$ cells regulatory (Tregs), neutrophils, plasma cells, mast cells activated, $\mathrm{T}$ cells cellular helper, T cells CD8, T cells CD4 memory resetting and macrophases M1 are core immune cells contributing to the progression of Alzheimer's disease.

Conclusion: The results of enrichment analysis and immunoosmotic analysis showed that immune pathways and immune cells played an important role in the occurrence and development of alzheimer disease. The selected key genes are used as biomarkers related to the pathogenesis of alzheimer disease to further explore the pathways and cells.

\section{INTRODUCTION}

Alzheimer's disease (alzheimer disease) is a degenerative disease that is prevalent among the elderly. In the past few decades, research in the field of alzheimer disease has increased significantly, driven by availability of the technology for isolating and identifying amyloid from the brain. ${ }^{1}$ Alzheimer's disease (alzheimer disease) is characterized by amyloid beta (A $\beta$ ) accumulation, tau pathology and neuroinflammation. Proinflammatory cytokines secreted by activated microglia may lead to sustained low-grade inflammation of neurons. The production of A $\beta$ and the pathway of neuroinflammation may jointly promote the pathology associated with alzheimer disease. Currently, the pathological features of amyloid plaque and neurofibrillary tangle are the main criteria for the diagnosis of alzheimer disease ${ }^{2}$.However, diagnosis of alzheimer disease through histopathology is invasive and can lead to brain injury. Presently, the diagnosis and treatment of alzheimer disease faces many challenges, such as lack of effective drugs and expensive diagnostic tests. In recent years, researchers have been committed to studying the biological processes driving alzheimer and identifying the abnormally expressed genes in the pathogenesis of alzheimer as new biomarkers. Identifying gene mutation sites associated with 
alzheimer disease is very important to explore the pathogenesis of alzheimer disease and further find therapeutic targets.

Weighted co-expression network analysis (WGCNA) is a powerful method for describing gene expression correlations using microarray databases. ${ }^{3}$ It is used to identify correlated genes (modules), analyze characteristic genes of the modules or hub genes determine modules of interest by based on correlation coefficients between modules and phenotypic traits, as well as explore the relationships between modules and traits. This approach facilitates can be used to identify therapeutic targets and biomarkers of various diseases. ${ }^{4}$ WGCNA is widely utilized to study diverse diseases such as diabetes $^{5}$, cancer ${ }^{6}$, brain imaging data analysis ${ }^{7}$, proteomic and metabolomic analyses. ${ }^{8}$

Previous studies have used WGCNA analysis to study Alzheimer's disease and screen gene markers. ${ }^{9}$ The difference between this study and previous studies is that through enrichment analysis and immune infiltration analysis, it is found that immune cells and immune infiltration are inseparable from the pathogenesis of Alzheimer's disease. In the current study, gene expression data sets obtained from integrated Gene Expression Omnibus database (GEO) were analyzed to identify differentially expressed genes (DEGs). Next, WGCNA analysis was adopted to find the core module. In addition, GO and KEGG enrichment analyses were carried out to reveal the roles of the core module, and construct a protein-protein interaction (PPI) network for proteins associated with the core module. Subsequently, the following hub genes, namely $A T P 2 A 2$, ATP6V1D, CAP2, SYNJ1 and GHITM. Logistic regression analysis and Receiver operating characteristics (ROC) was performed to explore the clinical value of each gene in diagnosis of Alzheimer. The genes in the core module are intersected with the hub genes, and four intersecting genes were selected. Further analysis was carried out on the four genes. The four genes were subjected to Gene set enrichment analysis (GSEA). Finally, CIBERSORT the tool was employed to investigate immune infiltration in alzheimer disease.

Keywords: Genetic biomarkers; Weight gene co-expression network analysis(WGCNA); Alzheimer's disease; epigenetic changes; immune infiltration. 


\section{Data collection and preprocessing}

The expression profile datasets were retrieved from the Gene Expression Omnibus database (GEO). GSE1297(22 Alzheimer samples and 9 normal control samples from hippocampal, Blalock EM et al., published 2004), GSE5281(10 Alzheimer samples, 13 normal control samples from entorhinal cortex; 10 Alzheimer samples, 13 normal control samples from hippocampus; 16 Alzheimer samples, 12 normal control samples from medial temporal gyrus; 9 Alzheimer samples, 13 normal control samples from posterior cingulate; 23 Alzheimer samples, 11 normal control samples from superior frontal gyrus and 19 Alzheimer samples, 12 normal control samples from primary visual cortex, Stephan DA et al., published 2006), GSE36980(15 Alzheimer samples, 18 normal control samples from frontal cortex; 10 Alzheimer samples, 19 normal control samples from temporal cortex; and 8 Alzheimer samples, 10 normal control samples from hippocampus, Nakabeppu Y et al., published 2013), GSE4226(14 Alzheimer samples, 14 normal control samples from peripheral blood mononuclear cell, Maes OC et al., published 2006) and GSE132903(97 Alzheimer samples, 98 normal control samples from temporal cortex, Piras IS et al., published 2019) datasets were selected for analysis in this study. GSE1297 dataset was used to explore relevant modules and hub genes, whereas the other datasets were used as validation sets. ${ }^{10}$ GSE1297 dataset was analyzed using GPL196 chip analyzer platform. ${ }^{11}$ Data on related clinical characteristics including age, disease status, PMI, NFT, BRAAK, MMSE and gender were also retrieved. All analyses were conducted in R (version 4.1.5) software. A flowchart showing the study design is presented in Figure 1.

\section{Analysis of differentially expressed genes}

According to the annotation platform of each expression profile, the probe is combined with the gene name to remove the empty probe. ${ }^{12}$ The lncRNA transcript was deleted from the dataset. A total of 4440 genes were selected to analysis of differentially expressed genes (DEGs). Analysis of DEGs was conducted using "limma" R package. Expression data of the gene matrix was $\log 2$ transformed. $P$-values were adjusted using the false discovery rate (FDR) method. Genes with abs $\mid \log 2$-fold change $\mid>=0.5$ and $\mathrm{P}$-value $<0.05$ were considered as DEGs. Upregulated and 
downregulated DEGs were selected based on $\mid \log 2$-fold change| and visualized as a volcano map using "ggplot2" R package.(Fig2A) DEGs were presented as a heatmap using "pheatmap" R package. $(\text { Fig2B })^{13}$

\section{Weighted gene co-expression network analysis (WGCNA)}

Further, 4440 genes were selected for WGCNA analysis. "WGCNA" R package was used for WGCNA analysis to explore modules related to clinical traits. All samples were included in the group, and network topology analysis was then performed with a threshold cut-off ranging from 1 to 20. Appropriate soft power threshold was selected for calculation of the adjacency matrix. A gene co-expression network was predicted with a power value equal to 5 (scale-free $\mathrm{R}^{\wedge 2}=0.85$ ), which showed a scale-free topology with complete module characteristics. A dendrogram was generated and similar clinical traits of the samples were explored to determine whether the 31 samples in the GSE1297 dataset were suitable for network analysis. The connectivity of the model was calculated, a connection matrix was constructed, and the number of modules adjusted. Soft power threshold adjacency matrices were then converted into a topological overlap matrix (TOM) and a TOM dissimilarity matrix (1-TOM). Genes with similar expression levels were clustered into several modules by hierarchical clustering function according to 1-TOM. Gene significance (GS) obtained from the $\log 10$ transformation of the $p$ value in a linear regression between the gene expression and clinical traits was computed to determine the significance of each module. Each module was represented by module eigengene (MEs), and the correlation coefficient between each module was calculated. Gene significance (GS) and module significance (MS) were used to quantify the expression configuration of modules related to sample types, form module traits, and for identification of modules closely related to the traits. WGCNA algorithm was used to determine the relationship between modular characteristic genes (MEs) and the clinical traits of alzheimer disease using Pearson correlation coefficient (PCC). The function cor P-value Student was used for determination of the Student asymptotic P-value. In addition to the gray module, the module with the highest or lowest correlation with alzheimer disease was selected as the key module $(P<0.05)$. The genes in the key modules were selected for subsequent analysis.

\section{Functional enrichment analysis and protein-protein interaction network (PPI) analysis}

Gene Ontology (GO) annotation and Kyoto Encyclopedia of genes and genomes (KEGG) enrichment analysis was performed using DAVID tool (http://david.abcc.ncifcrf.gov/) to explore 
141 the biological significance of selected module genes. GO terms and KEGG pathways were 142 visualized using the "ggplot" R package. The top 20 GO terms including biological process (BP), 143 cellular component (CC), and molecular function (MF) of the key modules, were presented in a 144 bar plot. In addition, the top 20 KEGG pathways of the key modules were presented as a bubble 145 chart.

146 Potential interactions among the proteins encoded by these genes were predicted using STRING webserver (version11.5; http://string-db.org). A combined interaction score $>0.5$ was considered

148 statistically significant. The minimum required interaction score was set at highest confidence 149 (0.900). The protein-protein interaction (PPI) network was visualized using Cytoscape tool $150 \quad$ (version 3.8.2).

\section{5. Network construction and identification of Hub genes}

152 The edge data results from WGCNA topology analysis were imported into Cytoscape, and a network was constructed according to the edge with weight $>0.7$. The top five genes in the network were selected using the plug-in unit "cytohubba" in Cytoscape software (version 3.6.2), according to 12 topology analysis algorithms and were presented as hub genes.

\section{Efficacy evaluation}

Logistic regression model was used to establish a prediction model. Receiver operating characteristics (ROC) curve analysis and the area under the curve (AUC) was performed using "ROCR" R package to explore the clinical value of each gene in diagnosis of Alzheimer. AUC value more than 0.7 indicated that the model had significant specificity and sensitivity. GSE5281, GSE36980, GSE4226 and GSE132903 datasets were used for external validation.

\section{Gene sets enrichment analysis (GSEA)}

Intersection genes between hub genes and key modules were selected for further analysis. A Venn diagram was generated using the "Draw Venn Diagram" webserver (http://bioinformatics.psb.ugent.be/webtools/Venn/).

Specific signaling pathways associated with these core genes and the potential molecular mechanisms in modulating progression of Alzheimer were explored. Gene Sets Enrichment Analysis (GSEA) (3.0.0 Edition, Broad College, MIT and University of California Board of Trustees) explored the possible biological functions of key genes. The "c2. cp.kegg.v3.0.symbols" function was used for enrichment analysis. The consistent $p$-value of each gene set was calculated 
and association between disease types and biological processes was explored through GSEA significant enrichment analysis. Terms with $P<0.05$ and $\mathrm{FDR}<0.25$ were defined as significant terms.

\section{Immune cell infiltration analysis}

CIBERSORT tool is used for analysis of the different immune cell types of tissues. CIBERSORT was adopted for analysis of the merged expression data and for determination of immune cell infiltrations to determine the relative proportions of infiltrating immune cells in Alzheimer. ${ }^{14}$ A $P<0.05$ was used to filter the samples. The percentage of each immune cell type in the samples was calculated and presented as a bar plot. A heat map of the 22 immune cells was generated using the "pheatmap" $\mathrm{R}$ package. A correlation heatmap showing the relationship of the 22 types of infiltrating immune cells was generated using "corrplot" package.

\section{RESULTS}

\section{DEGs were identified between normal and alzheimer disease samples}

A total of 4440 genes were selected after preprocessing. Moreover, 756 DEGs were identified between alzheimer disease and normal samples. Out of the 756 DEGs, 442 were upregulated and 314 were downregulated (Fig. 2A). The top 100 DEGs (selected according to $|\log 2 \mathrm{FC}|$, including the top 50 upregulated genes and the top 50 downregulated genes) were selected to determination of expression patterns. A heatmap showing clustering of DEGs is presented in Figure 2B. The results showed that the Alzheimer samples and the control samples could be significantly distinguished according to the characteristics of the DEGs.

\section{Gene co-expression modules were identified}

WGCNA analysis was performed on the expression data of 4440 mRNAs in GSE1297 dataset. The "picksoft Threshold" function in WGCNA software package was used to filter out power parameters in the range of $1-20$. The soft threshold power $\beta$ was to 5 (scale-free $\mathrm{R}^{\wedge 2}=0.85$ ) to obtain a scale-free network (Fig. 3A, B). Reliability of the scale-free topology was further analyzed according to the soft threshold equal to 5 (Fig. 3C). The cut-off height was set to 0.2 to merge similar modules in the clustering tree resulting in nine modules (Fig. 4A). The Topological Overlap Matrix $(T O M)$ and dissTOM $=1-T O M$ were obtained with non-clustering DEGs in the gray module (Fig. 4B). MEs were used as illustrative profiles and module correspondence was 
computed by eigengene correlation. Interaction relations of the 9 modules was determined, and a network heatmap was generated (Fig. 4C). The heatmap of module trait correlation showed that the most significant correlation feature was in the blue module, which had a significant negative correlation with MMSE score (correlation coefficient $=-0.52, P=0.002$; Fig. 4D). This finding indicated that the blue module (correlation coefficient $=0.44, P=0.01$; Fig. 4C) was the key module related to alzheimer disease disease status.

3. Genes related to alzheimer disease were significantly enriched and PPI network analysis

\section{of the key module}

Genes in the blue module were selected for further analysis. A total of 76 genes were identified in the blue module. GO and KEGG analyses were performed to explore the potential biological functions of genes in the key module. The top 20 significantly enriched GO terms were retrieved from GO functional annotation (Fig. 5A). Functional enrichment analysis indicated that genes in key modules were mainly involved in biological process (BP) terms, including: positive regulation of heart rate, glutamate secretion, regulation of macroautophagy, neurotransmitter secretion, regulation of insulin secretion, ion transmembrane transport and chemical synaptic transmission. The cell component (CC) showed that genes were significantly enriched in: calcineurin complex, myelin sheath, growth cone, postsynaptic density, neuron projection, neuronal cell body, cell junction, perinuclear region of cytoplasm, membrane and extracellular exosome. Molecular function (MF) analysis showed that the genes were mainly enriched in: calcium-dependent protein interacting with the blue module were mainly enriched in: synaptic vesicle cycle, cGMP-PKG signaling pathway, glutamatergic synapse, pathways of neurodegeneration - multiple diseases, and Alzheimer disease. The top 20 KEGG pathways were selected (Fig. 5C). The interaction network for the top 20 KEGG pathway is displayed in Figure 5D. GO and KEGG analyses showed that the genes in the key module were enriched in the process of differentiation and growth of nerve cells and neurotransmitter transmission, and some of them were involved in pathogenesis of neurodegenerative diseases or Parkinson's disease.

Genes from the blue module were selected for further analysis. A PPI network composed of 76 nodes and 28 edges was constructed using the STRING tool(https://cn.string-db.org/) to further 
determine their association at the protein level. The PPI network was visualized using Cytoscape software (Fig. 5E). The network status was as follows: average node degree $=0.737$; expected number of edges $=9$.

\section{Hub genes were identified}

Edge data were obtained from weight gene co-expression network analysis. The data were imported into Cytoscape software, and the weight was set at $>0.7$ for construction of the network

(Fig. 6). Cytohubba plug-in in Cytoscape tool was used to comprehensively explore the results of 12 algorithms. The top five genes were selected as the hub genes, and included $A T P 2 A 2$, ATP6V1D, CAP2, SYNJ1 and GHITM .

\section{Efficacy evaluation of hub genes}

Logistic regression model was used to establish a prediction model. The regression equation was as follows: $\mathrm{Y}=62.353420+3.620291 *$ ATP2A2-16.089225*ATP6V1D $+2.823338 *$ CAP2 $+2.035213 *$ SYNJ1 $+1.501149 *$ GHITM. ROC analysis of the prediction model composed of five hub genes was performed using GSE1297 dataset as the test set, and AUC value of 0.9192 was obtained. The model was further validated using the verification set (GSE5281, GSE36980, GSE4226 and GSE132903), and the AUC was 0.8427, 0.8162, 0.801 and 0.799, respectively. All AUC values in the test set and validation sets were above 0.7, indicating that the model has high sensitivity and specificity. In addition, this finding implies that the five hub genes are potential biomarkers for detection of Alzheimer (Fig. 7).

Samples of GSE1297 test dataset were obtained from the hippocampus, and the samples of posterior cingulate gyrus, superior frontal gyrus and primary visual cortex. GSE36980 test set samples were obtained from frontal cortex, temporal cortex and hippocampus. GSE4226 test set samples were obtained from peripheral blood mononuclear cell. GSE132903 test set samples were

\section{GSEA reveals potential biological functions}

Take the intersection of hub gene and blue module gene, obtain four cross genes, which are ATP6V1D, CAP2, SYNJ1 and ATP2A2, as shown in Venn diagram(Fig. 8). The intersection genes were further analyzed. 
Further analysis of biological pathways indicated that hub genes are implicated in immune, inflammatory and other metabolic processes. The samples in GSE1297 dataset were assigned to "high expression group" and "low expression group" according to the expression levels of $A T P 2 A 2, A T P 6 V 1 D, C A P 2$ and $S Y N J 1$ genes. The four hub genes corresponded to 10, 8, 9 and 8 enrichment pathways in the high expression group, respectively.

GSEA results showed that cholera infection, oxidative phosphorylation, citrate cycle, epithelial cell signaling in helicobacter pylori infection, alanine and aspartate metabolism, ubiquitin mediated proteolysis, RNA polymerase, glutamate metabolism, proteasome, selenoamino acid metabolism and pyruvate metabolism pathways were highly enriched in the high expression of hub gene group $(P<0.05)$.(Fig. 9) Immune infection pathways such as cholera infection and Helicobacter pylori infection play a key role in the high expression of alzheimer disease. The number of pathways associated with low expression of the genes was significantly low mainly included: porphyrin and chlorophyll metabolism, cell communication, cytokine-cytokine receptor interaction, metabolism of xenobiotics by cytochrome p450 and maturity onset diabetes of the young. Further studies should conduct detailed analysis of core gene enrichment pathway to explore pathogenesis of alzheimer disease, and the specific mechanism should be verified through in vitro and in vivo studies.

\section{Immune cell infiltration analysis}

CIBERSORT algorithm was used to predict infiltration of the immune cells between patients with Alzheimer and the control group. The percentage of 22 immune cells in each sample was presented in a bar graph (Fig. 10A). The samples with significant immune infiltration $(P<0.05)$ were selected and a percentage heat map of immune cells was generated (Fig. 10B). Correlation analysis of 22 immune cells showed that infiltrations of $\mathrm{T}$ cells regulatory (Tregs) was positively correlated with that of neutrophils $(r=0.87)$, plasma cells were positively correlated with activated mast cells $(r=0.84), T$ follicular helper cells were negatively correlated with CD8 $\mathrm{T}$ cells $(r=-0.95)$, memory resting CD4 T cells were negatively correlated with M1 macrophages ( $\mathrm{r}$ $=-0.92)$ and $\mathrm{CD} 8 \mathrm{~T}$ cells were negatively correlated with M1 macrophages $(\mathrm{r}=-0.82)($ Fig. 10C). A significant difference in immune cell infiltration was observed between brain tissue of alzheimer disease patients and normal brain tissue. These finding indicates that $\mathrm{T}$ cells regulatory (Tregs), neutrophils, plasma cells, activated mast cells, T follicular helper cells, CD8 T cells, CD4 memory 
resetting $\mathrm{T}$ cells and M1 macrophages are potential core immune cells involved in promoting progression of alzheimer disease.

\section{DISCUSSION}

Alzheimer's disease (alzheimer disease) is the primary cause of dementia among the elderly. Clinically, it mainly manifests as progressive impairment of memory and other cognitive areas. Pathologically, the initiation of alzheimer disease is driven by genetic factors, environmental factors and ageing.The major pathological changes in alzheimer disease include: neuron loss and brain atrophy, synaptic failure, neurofibrillary tangles, and $\beta$-amyloid (A $\beta)$ plaques. ${ }^{15}$ So far, the diagnosis of alzheimer disease is still based on the pathological changes of amyloid protein and tau biomarkers found in postmortem brain tissue sampling, there is no clear diagnostic criteria and targeted treatment for alzheimer disease when Alzheimer's disease patients are alive. ${ }^{16-17}$ Therefore, it is crucial to search for genetic biomarkers and potential mechanisms of alzheimer disease which can be potential treatment targets. ${ }^{18}$

In this study, data was mined from gene chips extracted from different brain regions of alzheimer disease patients and normal people through autopsy. WGCNA analysis was carried out to explore changes in gene expression between alzheimer disease and normal people. Compared with other traditional differential expression analysis methods, WGCNA has many advantages. For instance, it analyses co-expression patterns and reveals functional modules containing related genes. The hub genes in the modules can be used as detection biomarkers or treatment targets.

We identified nine co-expression modules via dynamic tree cutting, from which the blue module was found to be the key module $(\mathrm{P}<0.01)$. Subsequently, GO KEGG and PPI analyses were carried out for the genes in the blue module. A network was constructed and used to reveal core genes. Five hub genes: ATP2A2, ATP6V1D, CAP2, SYNJ1 and GHITM were identified. It has been previously reported that the sarco / endoplasmic reticulum (SR / ER) Ca $\left(2^{+}\right)$- ATPase (SERCA) has an important role in $\mathrm{Ca}^{2+}$ regulation. Impaired SERCA activity may lead to various diseases such as alzheimer disease, diabetes, heart failure, and cancer. ${ }^{19}$ Overexpression of ATP2A2 correlated with good prognosis of patients with diffuse astrocytic tumors. ${ }^{20}$ Mutations in ATP2A2 are thought to cause dyskeratosis and abnormal intercellular adhesion. ${ }^{21}$ Previous 
literature reported that, ATP6V1D participates in the transport of hydrogen ion and can lead to cancer development.$^{22}$ ATP6V1D is also a central component of vascular ATPase. ${ }^{23}$ Srivastav's team found that CAP2 / hap43 regulates the transcription of various genes and possess many conserved protein domains. ${ }^{24}$ Adenylate cyclase associated protein 2 (CAP2) is involved in the regulation of cellular actin dynamics. Previous studies have linked this protein to tumor progression. However, its expression level in Alzheimer's disease has not been evaluated. ${ }^{25}$ Previous studies have confirmed that Synaptojanin 1 (SYNJ1) is a brain-enriched lipid phosphatase, which mainly participates in autophagosome/endosome transport, synaptic vesicle alzheimer disease. Moreover, SYNJ1 is related to amyloid-induced toxicity. However, the upregulated in neurofibrillary tangles (NFTs), plaque associated dystrophic axons and Hirano bodies. SYNJ1 immunoreactivity is elevated in neurons and senile plaques in alzheimer disease patients with one or two apolipoprotein (APOE) $\varepsilon 4$ alleles. ${ }^{26}$ Phosphatidylinositol phosphatase synaptic binding protein 1 (SYNJ1), which regulates synaptic function, has been found to be negatively related to expression of synaptophysin, in postmortem brains of adult patients with Professor Miranda, SYNJ1 overexpressing mice showed age-related cognitive decline to the level of human sporadic alzheimer disease, which is caused by hippocampal hyperexcitability and spatial reproducibility deficits in the position field. In all types of alzheimer disease, SYNJ1 contributes to memory deficits in the aging hippocampus. ${ }^{27}$ Professor George's findings on synaptic connexin have shown that synaptic cell protein 1(SYNJ1) is involved in alzheimer disease and Parkinson's disease. ${ }^{28}$ SYNJ1 downregulation protects against amyloid beta peptide (A $\beta$ )-induced toxicity in alzheimer disease, Professor Drouet's research on synaptic connexin 1 mutation in Parkinson's disease confirms this view. ${ }^{29}$ Growth hormone-induced transmembrane protein (GHITM) is a highly conserved transmembrane protein. ${ }^{30}$ The Transmembrane Bax interactivator -1 Motif (TMBIM) protein family is evolutionarily conserved and associated with susceptibility to cell death. The only member of this protein expressed in mitochondria is TMBIM5 (also known as GHITM or MICS1). Professor seitaj's team found that this protein 
regulates crest tissue and is linked to $\mathrm{CHCHD} 2$, a protein in mitochondrial intima that plays a role in Parkinson's disease. ${ }^{31}$ In summary, the five key / hub genes we screened are likely to be biomarkers and treatment targets in Alzheimer's disease.

The logistic regression model was constructed and applied to perform comprehensive evaluation of their ability to predict alzheimer disease, and to find other alzheimer disease datasets (gene expression datasets extracted in other regions of the cerebral cortex) to validate the diagnostic value of the model. alzheimer disease, such as oxidative phosphorylation, citric acid cycle, and also including immune infection pathways such as cholera infection and Helicobacter pylori infection. This study found that phosphorylation pathway involved in the pathogenesis of alzheimer disease through data mining. The brain requires continuous energy supply in the form of ATP, most of which is produced by glucose through oxidative phosphorylation in the mitochondria, supplemented by aerobic glycolysis in the cytoplasm. Phosphorylation pathway and abnormal glucose metabolism complement each other. Abnormal glucose metabolism is also closely related to the pathogenesis altered in the brain of preclinical alzheimer disease patients. ${ }^{34}$ We also enriched the oxidative of Alzheimer's disease. Cunnane's team found that in elderly patients with neurodegenerative diseases, brain glucose metabolism is impaired in a region-specific, and disease-specific manner-this problem precedes the onset of symptoms in alzheimer disease. ${ }^{32}$ Professor Pawlosky and others found that in alzheimer disease patients and alzheimer disease mouse models, hypoglycemia metabolism in the brain precedes memory loss and cognitive decline, and ketone metabolism in the brain corrects some defects related to glucose hypometabolism through glycolysis. ${ }^{33}$ Our study found that citric acid cycle is involved in the pathogenesis of Alzheimer's disease by mining the database, which supports these previous studies. Professor Butterfield's team found that oxidative stress contributes to the pathogenesis and progression of alzheimer disease and its early amnestic mild cognitive impairment (aMCI). Some of these pathways are stress pathway through data mining, which is consistent with previous studies. Immune infection pathway is also involved in the pathogenesis of Alzheimer's disease. Previous Professor Nikolic 
found that cholera toxin and cholera infection pathways may be involved in the occurrence of

alzheimer disease. ${ }^{35}$ During senescence and neurodegeneration, activation of the

381 innate immune system can occur. ${ }^{36}$ Microglia are innate immune cells in the central nervous system of patients with alzheimer disease. Proinflammatory cytokines secreted by activated microglia may lead to a positive feedback loop between neurons and microglia, resulting in sustained low-level inflammation. ${ }^{37}$ However, there are still many gaps in the study of immune infiltration pathways and the pathogenesis of Alzheimer's disease.

In summary, WGCNA was adopted in the present study to explore co-expressed gene modules and hub genes in alzheimer disease. The biological functions of selected core modules were determined, as well as the pathways associated with the hub genes. The relationship between hub genes and immune infiltration was also explored. These results can be used to explore biomarkers of alzheimer disease.

391 Nevertheless, this study has some limitations. First of all, alzheimer disease data sets in GEO database are not as large and recognized as tumor data sets. Therefore, more data sets should be combined for analysis and verification as much as possible to ensure the accuracy of the results. Secondly, in GEO database, alzheimer disease has less clinical information, so there is less information that can be used for data analysis. Third, the genes screened in this study are only limited to the data analysis stage. It is necessary to verify the results of data analysis through in vivo, in vitro and clinical experiments, whether the screened genes are different between the diseased group and the normal group, and whether the difference is statistically significant.

In conclusion, the WGCNA method, combined with clinical information, were applied to screen for key modules in alzheimer disease, and analyze the biological roles of genes in the modules. Hub genes were screened and used to construct a prediction model. Performance of the prediction model was verified via test set and verification sets. The intersection genes between hub genes and genes of the core module were subjected to GSEA which revealed crucial enrichment pathway of the genes in alzheimer disease. Finally, results of immune infiltration showed that $\mathrm{T}$ cells regulatory (Tregs), neutrophils, plasma cells, mast cells activated, $\mathrm{T}$ cells cellular helper, $\mathrm{T}$ cells the progression of Alzheimer's disease. 


\section{Ethics approval and consent to participate:}

$411 \quad$ Not applicable.

\section{Consent for publication:}

413 Not applicable.

\section{Availability of data and materials:}

415 The data used and analyzed during the current study are available from GEO

416 (https://www.ncbi.nlm.nih.gov/geo/).

\section{Competing interests:}

418 The authors declare that they have no competing interests.

\section{Funding:}

420 the National Natural Science Foundation of China (Grant No. 92163213 and 81970085) and the

421 Tianjin science and Technology plan project (Grant No. 17ZXMFSY00080).

\section{Authors' contributions:}

423 KeFei Duan, Qiang Zhang and Jin Tan conceived and designed the article. KeFei Duan wrote the 424 manuscript. Yuyang Miao contributed to the language modification and guidance. All authors read 425 and approved the final manuscript.

\section{Acknowledgements:}

$427 \quad$ Not applicable.

\section{REFERENCES}

1 Hane FT, Robinson M, Lee BY, Bai O, Leonenko Z, Albert MS. Recent Progress in Alzheimer's Disease Research, Part 3: Diagnosis and Treatment 2017;57(3):645-665.

2 DeTure MA, Dickson DW. The neuropathological diagnosis of Alzheimer's disease. Mol Neurodegener. 2019 Aug 2;14(1):32.

3 Langfelder P, Horvath S. WGCNA: an R package for weighted correlation network analysis. BMC Bioinformatics. 2008 Dec 29;9:559.

4 Liang JW, Fang ZY, Huang Y, Liuyang ZY, Zhang XL, Wang JL, Wei H, Wang JZ, Wang XC, Zeng J, Liu R. Application of Weighted Gene Co-Expression Network Analysis to Explore the Key Genes in Alzheimer's Disease. J Alzheimers Dis. 2018;65(4):1353-1364.

5 Zhu $Y$, Ding X, Xie Z, White X, Ni Z, King F, King F, Geng X.The symbiont pathogenesis of Alzheimer's disease and type 2 diabetes was explored by coexpression network analysis.Kr Alzheimer's Res.2020:17(6):566-575.

6 Tian Z, He W, Tang J, Liao X, Yang Q, Wu Y, Wu G. Identification of Important Modules and Biomarkers in Breast Cancer Based on WGCNA. Onco Targets Ther. 2020 Jul 12;13:6805-6817.

7 Heiland DH, Simon-Gabriel CP, Demerath T, Haaker G, Pfeifer D, Kellner E, Kiselev VG, Staszewski O, Urbach H, Weyerbrock A, Mader I. Integrative Diffusion-Weighted Imaging and Radiogenomic Network Analysis of Glioblastoma multiforme. Sci Rep. 2017 Mar 7;7:43523.

8 Pei G, Chen L, Zhang W. WGCNA Application to Proteomic and Metabolomic Data Analysis. Methods Enzymol. 2017;585:135-158.

9 Liang JW, Fang ZY, Huang Y, Liuyang ZY, Zhang XL, Wang JL, Wei H, Wang JZ, Wang XC, Zeng J, Liu R. Application of Weighted Gene Co-Expression Network Analysis to Explore the Key Genes in Alzheimer's Disease. J Alzheimers Dis. 2018;65(4):1353-1364.

10 Zhang Z, Liu Y, Wang W, Xing Y, Jiang N, Zhang H, Zhang H, He L, Yue W, Jiang L, Wang K. Identification of Differentially Expressed Genes Associated with Lymph Node Tuberculosis by the Bioinformatic Analysis Based on a Microarray. J Comput Biol. 2020 Jan;27(1):121-130.

11 Soleimani Zakeri NS, Pashazadeh S, MotieGhader H. Gene biomarker discovery at different stages of Alzheimer using gene co-expression network approach. Sci Rep. 2020 Jul 22;10(1):12210.

12 Sun Y, Lin J, Zhang L. The application of weighted gene co-expression network analysis in identifying key modules and hub genes associated with disease 
status in Alzheimer's disease. Ann Transl Med. 2019 Dec;7(24):800.

13 Zhao X, Yao H, Li X. Unearthing of Key Genes Driving the Pathogenesis of Alzheimer's Disease via Bioinformatics. Front Genet. 2021 Apr 16;12:641100.

14 Sui S, An X, Xu C, Li Z, Hua Y, Huang G, Sui S, Long Q, Sui Y, Xiong Y, Ntim M, Guo W, Chen M, Deng W, Xiao X, Li M. An immune cell infiltration-based immune score model predicts prognosis and chemotherapy effects in breast cancer. Theranostics. 2020 Oct 25;10(26):11938-11949.

15 Panza F, Lozupone M, Logroscino G, Imbimbo BP. A critical appraisal of amyloid- $\beta$-targeting therapies for Alzheimer disease. Nat Rev Neurol. 2019 Feb;15(2):73-88

16 Liu Z, Li H, Pan S. Discovery and Validation of Key Biomarkers Based on Immune Infiltrates in Alzheimer's Disease. Front Genet. 2021 Jul 1;12:658323.

17 Tan CC, Yu JT, Tan L. Biomarkers for preclinical Alzheimer's disease. J Alzheimers Dis. 2014;42(4):1051-69.

18 Cummings JL, Tong G, Ballard C. Treatment Combinations for Alzheimer's Disease: Current and Future Pharmacotherapy Options. J Alzheimers Dis. 2019;67(3):779-794.

19 Satoh K, Matsu-Ura T, Enomoto M, Nakamura H, Michikawa T, Mikoshiba K. Highly cooperative dependence of sarco/endoplasmic reticulum calcium ATPase SERCA2a pump activity on cytosolic calcium in living cells. J Biol Chem. 2011 Jun 10;286(23):20591-9.

20 Li WQ, Zhong NZ, He J, Li YM, Hou LJ, Liu HM, Xia CY, Wang LZ, Lu YC. High ATP2A2 expression correlates with better prognosis of diffuse astrocytic tumor patients. Oncol Rep. 2017 May;37(5):2865-2874.

21 Noda K, Takeichi T, Okuno Y, Takama H, Miura S, Kagami S, Hino H, Nakamura Y, Fujio Y, Konohana I, Otani A, Mukai H, Sugiura K, Akiyama M. Novel and recurrent ATP2A2 mutations in Japanese patients with Darier's disease. Nagoya J Med Sci. 2016 Dec;78(4):485-492.

22 Li X, Li H, Yang C, Liu L, Deng S, Li M. Comprehensive Analysis of ATP6V1s Family Members in Renal Clear Cell Carcinoma With Prognostic Values. Front Oncol. 2020 Oct 30;10:567970.

23 Göttle P, Förster M, Gruchot J, Kremer D, Hartung HP, Perron H, Küry P. Rescuing the negative impact of human endogenous retrovirus envelope protein on oligodendroglial differentiation and myelination. Glia. 2019 Jan;67(1):160-170.

24 Srivastav MK, Agarwal N, Natarajan K. Multiple Evolutionarily Conserved Domains of Cap2 Are Required for Promoter Recruitment and Iron Homeostasis Gene Regulation. mSphere. 2018 Aug 1;3(4):e00370-18.

25 Saker Z, Bahmad HF, Fares Y, Al Najjar Z, Saad M, Harati H, Nabha S. Prognostic impact of adenylyl cyclase-associated protein 2 (CAP2) in glioma: A clinicopathological study. Heliyon. 2020 Jan 18;6(1):e03236.

26 Ando K, Ndjim M, Turbant S, Fontaine G, Pregoni G, Dauphinot L, Yilmaz Z, Suain V, Mansour S, Authelet M, De Dekker R, Leroy K, Delatour B; Brain Bank NeuroCEB Neuropathology Network, Duyckaerts C, Potier MC, Brion JP. The lipid phosphatase Synaptojanin 1 undergoes a significant alteration in expression and solubility and is associated with brain lesions in Alzheimer's disease. Acta Neuropathol Commun. 2020 Jun 3;8(1):79.

27 Miranda AM, Herman M, Cheng R, Nahmani E, Barrett G, Micevska E, Fontaine G, Potier MC, Head E, Schmitt FA, Lott IT, Jiménez-Velázquez IZ, Antonarakis SE, Di Paolo G, Lee JH, Hussaini SA, Marquer C. Excess Synaptojanin 1 Contributes to Place Cell Dysfunction and Memory Deficits in the Aging Hippocampus in Three Types of Alzheimer's Disease. Cell Rep. 2018 Jun 5;23(10):2967-2975.

28 George AA, Hayden S, Stanton GR, Brockerhoff SE. Arf6 and the 5'phosphatase of Synaptojanin 1 regulate autophagy in cone photoreceptors. Inside Cell. 2016 Apr;1(2):117-133.

29 Drouet V, Lesage S. Synaptojanin 1 mutation in Parkinson's disease brings further insight into the neuropathological mechanisms. Biomed Res Int. 2014;2014:289728.

30 Yu W, Zhang B, Song H, Zhan R, Li L, He C, Jiang Q, Wang X, Wei L, Zhao N, Guo W, Wang X. Preliminary investigation demonstrating the GHITM gene probably involved in apoptosis and growth of the golden apple snail (Pomacea canaliculata). BMC Genomics. 2020 Jan 6;21(1):19.

31 Seitaj B, Maull F, Zhang L, Wüllner V, Wolf C, Schippers P, La Rovere R, Distler U, Tenzer S, Parys JB, Bultynck G, Methner A. Transmembrane BAX Inhibitor-1 Motif Containing Protein 5 (TMBIM5) Sustains Mitochondrial Structure, Shape, and Function by Impacting the Mitochondrial Protein Synthesis Machinery. Cells. 2020 Sep 23;9(10):2147.

32 Cunnane SC, Trushina E, Morland C, Prigione A, Casadesus G, Andrews ZB, Beal MF, Bergersen LH, Brinton RD, de la Monte S, Eckert A, Harvey J, Jeggo R, Jhamandas JH, Kann O, la Cour CM, Martin WF, Mithieux G, Moreira PI, Murphy MP, Nave KA, Nuriel T, Oliet SHR, Saudou F, Mattson MP, Swerdlow RH, Millan MJ. Brain energy rescue: an emerging therapeutic concept for neurodegenerative disorders of ageing. Nat Rev Drug Discov. 2020 Sep;19(9):609-633.

33 Pawlosky RJ, Kemper MF, Kashiwaya Y, King MT, Mattson MP, Veech RL. Effects of a dietary ketone ester on hippocampal glycolytic and tricarboxylic acid cycle intermediates and amino acids in a 3xTgalzheimer disease mouse model of Alzheimer's disease. J Neurochem. 2017 Apr;141(2):195-207.

34 Butterfield DA, Boyd-Kimball D. Oxidative Stress, Amyloid- $\beta$ Peptide, and Altered Key Molecular Pathways in the Pathogenesis and Progression of Alzheimer's Disease. J Alzheimers Dis. 2018;62(3):1345-1367.

35 Nikolic WV, Bai Y, Obregon D, Hou H, Mori T, Zeng J, Ehrhart J, Shytle RD, Giunta B, Morgan D, Town T, Tan J. Transcutaneous beta-amyloid immunization reduces cerebral beta-amyloid deposits without T cell infiltration and microhemorrhage. Proc Natl Acad Sci U S A. 2007 Feb 13;104(7):2507-12.

36 Heneka MT, McManus RM, Latz E. Inflammasome signalling in brain function and neurodegenerative disease. Nat Rev Neurosci. 2018 Oct;19(10):610-621.

37 Webers A, Heneka MT, Gleeson PA. The role of innate immune responses and neuroinflammation in amyloid accumulation and progression of Alzheimer's disease. Immunol Cell Biol. 2020 Jan;98(1):28-41. 


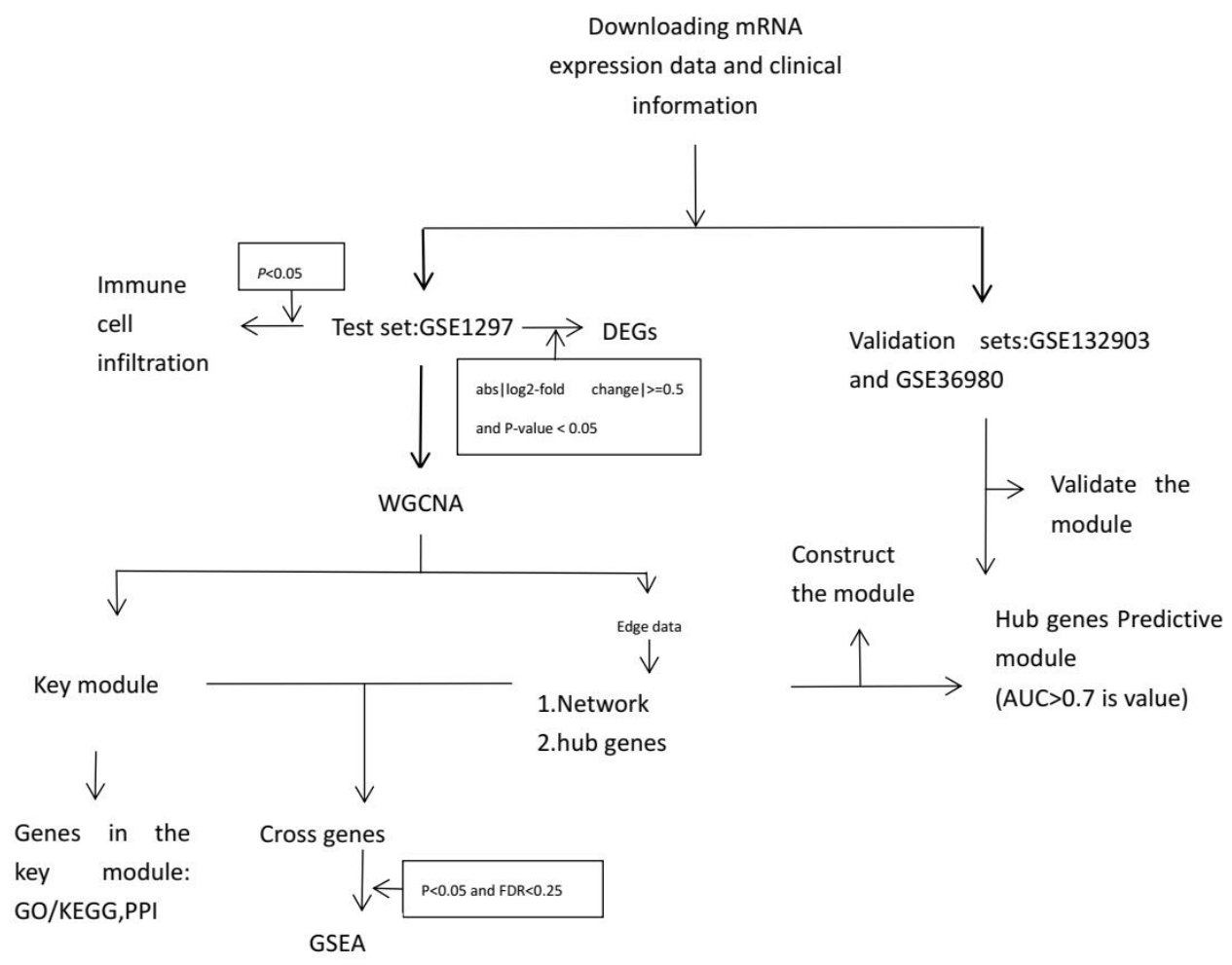

FIGURE 1| Flowchart of the Study. Abbreviation: DEGs, differential expression genes; WGCNA, weighted co-expression network analysis; GO, Gene Ontology; KEGG, Kyoto Encyclopedia of Genes and Genomes; PPI, protein-protein interaction; GSEA, Gene Sets Enrichment Analysis.

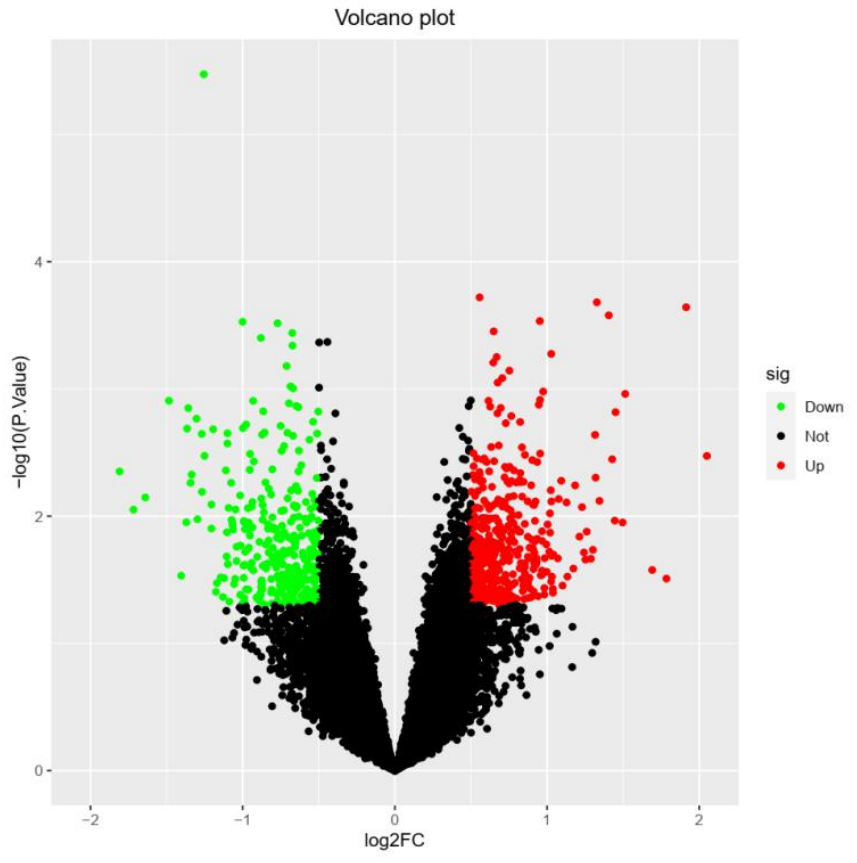




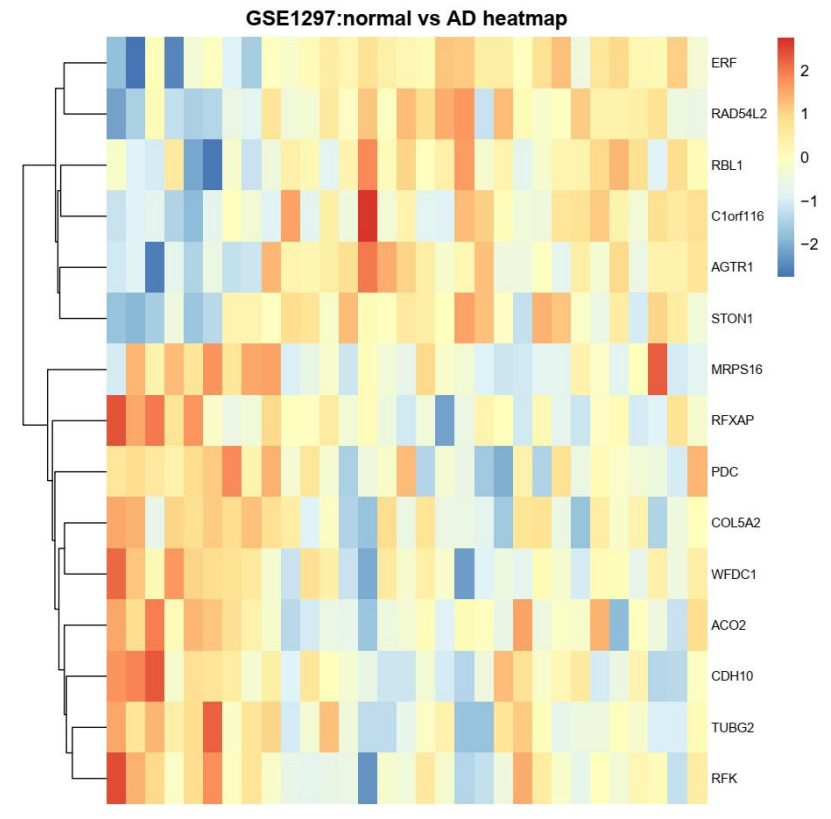

(B)

FIGURE 2 |Differentially expressed RNAs.

(A) A volcano plot showing the expression level of 4440 mRNAs. Green, and red dots represent the top 50 down-regulated and up-regulated mRNAs, respectively. Black dots represent not differentially expressed.

(B) Heat map clustering of the differentially expressed genes between alzheimer disease and normal samples.

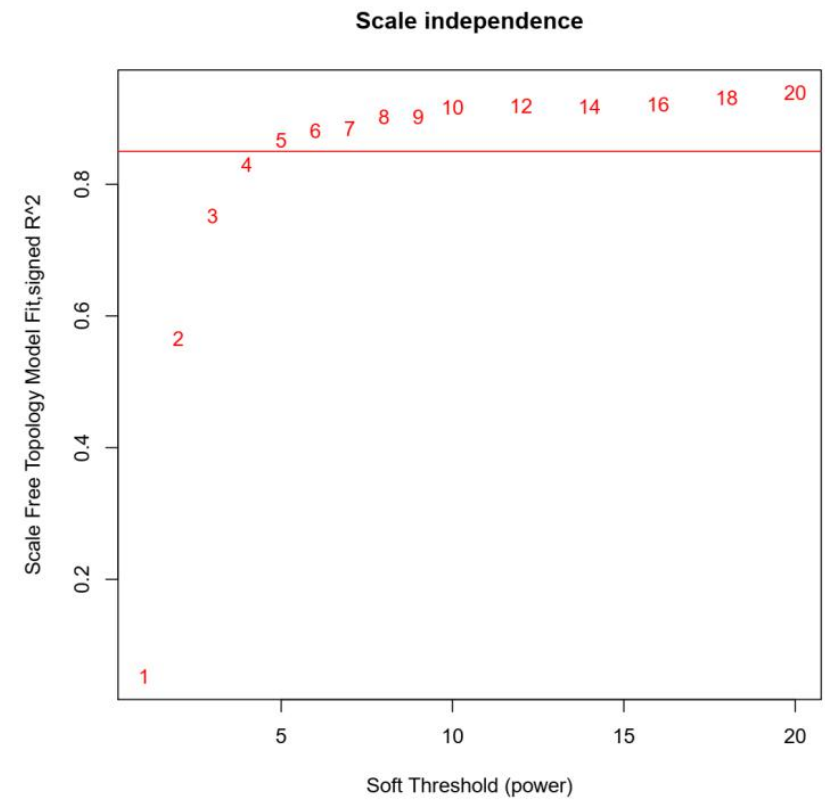




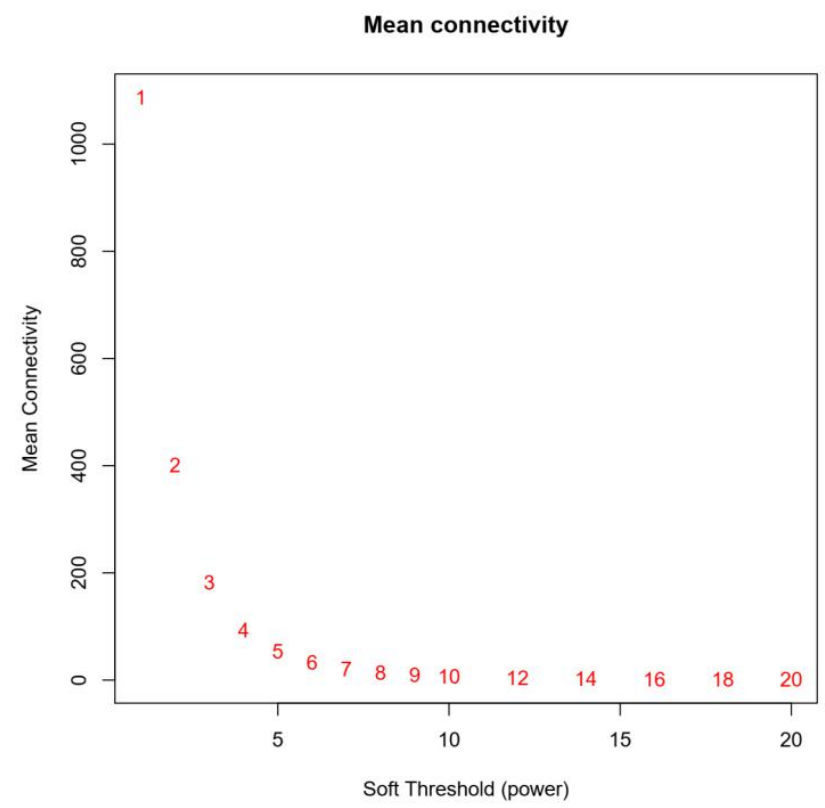

(B)

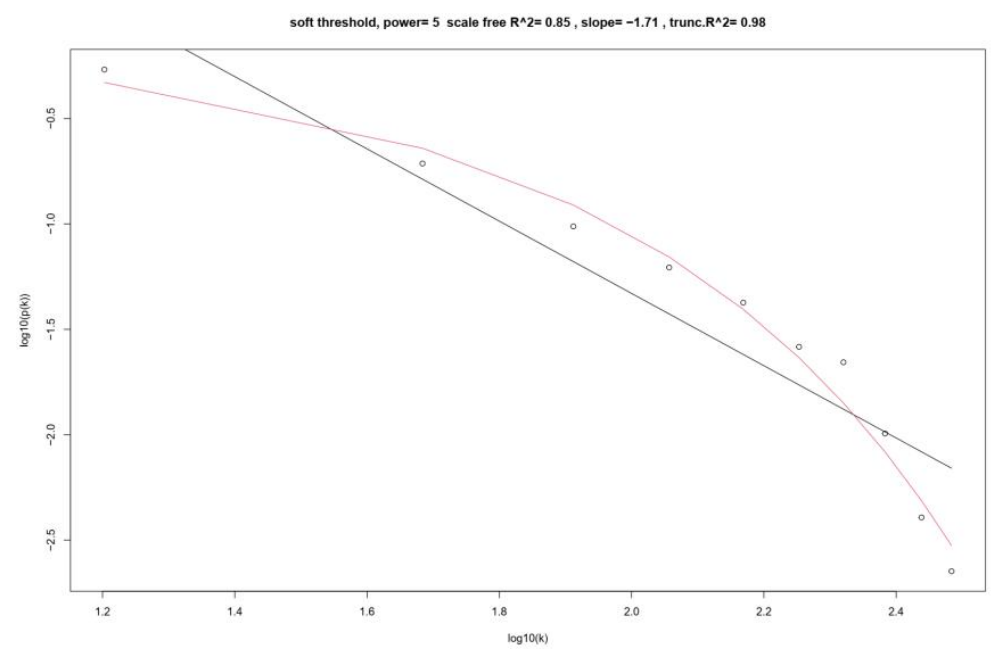

(C)

FIGURE 3|Scale independence and Mean connectivity

$\mathrm{R}^{\wedge 2}$ shows the scale independence measure. The mean connectivity value used for determination of optimal value of the soft threshold is shown, which ensures a scale-free network and retains important correlations. (A) Analysis of the scale-free fit index for various soft thresholds, with the soft threshold set at 5. (B) Analysis of the mean connectivity for various soft thresholds. (C) Analysis of the scale-free topology when the soft threshold is 5. 

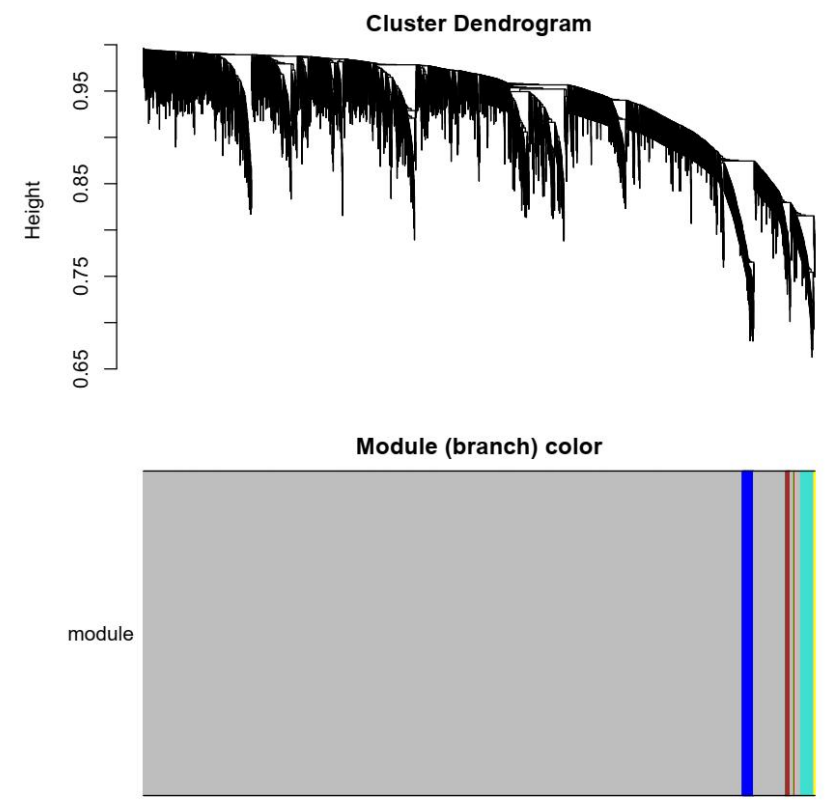

(A)

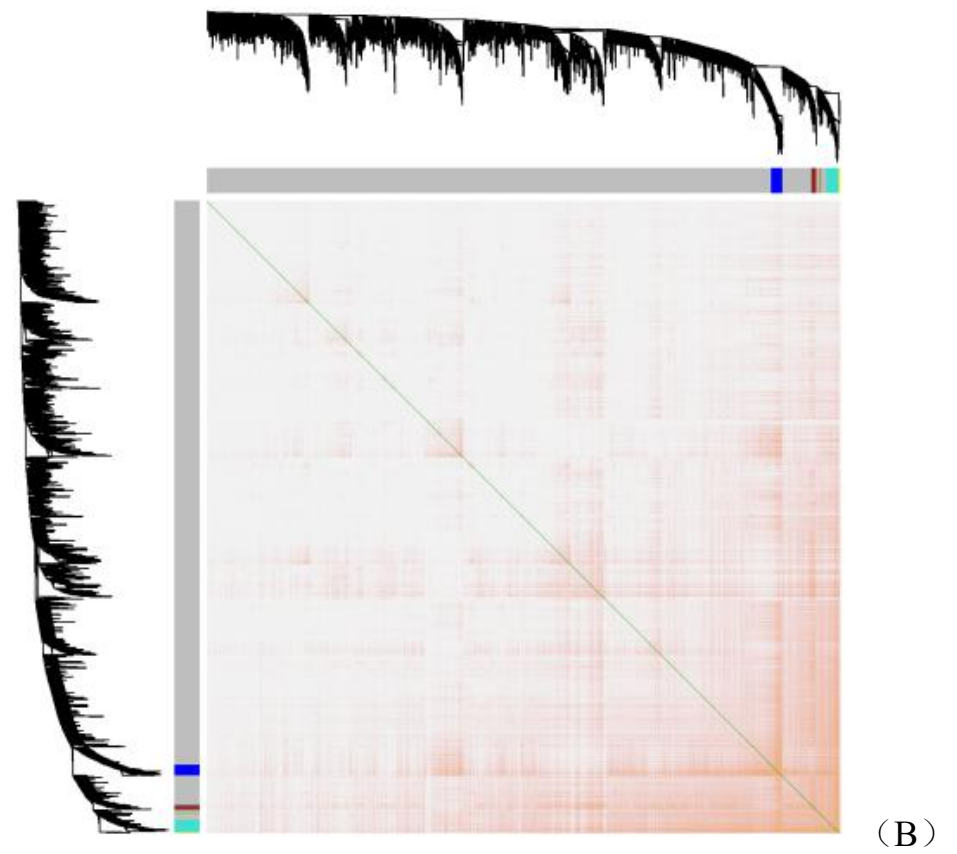




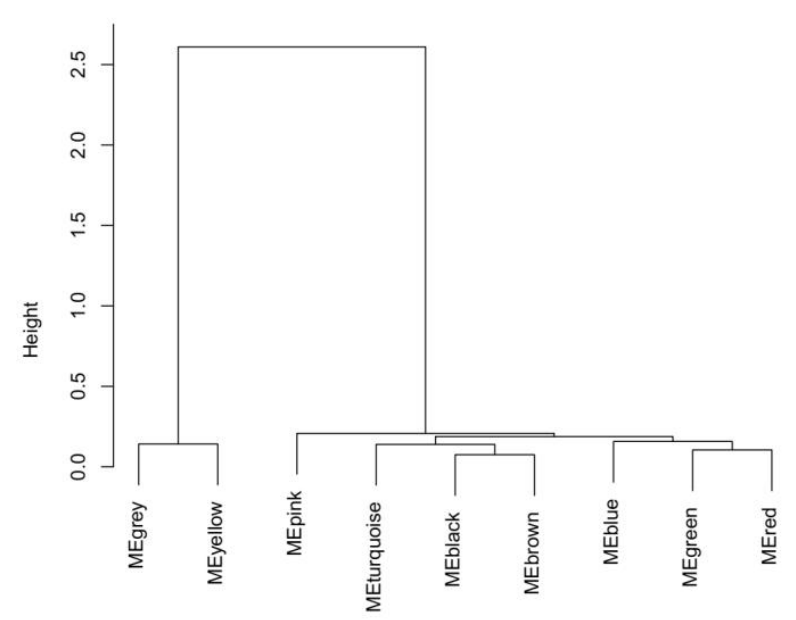

$\operatorname{dist(dissimME)}$

hclust (", "average")

(C)

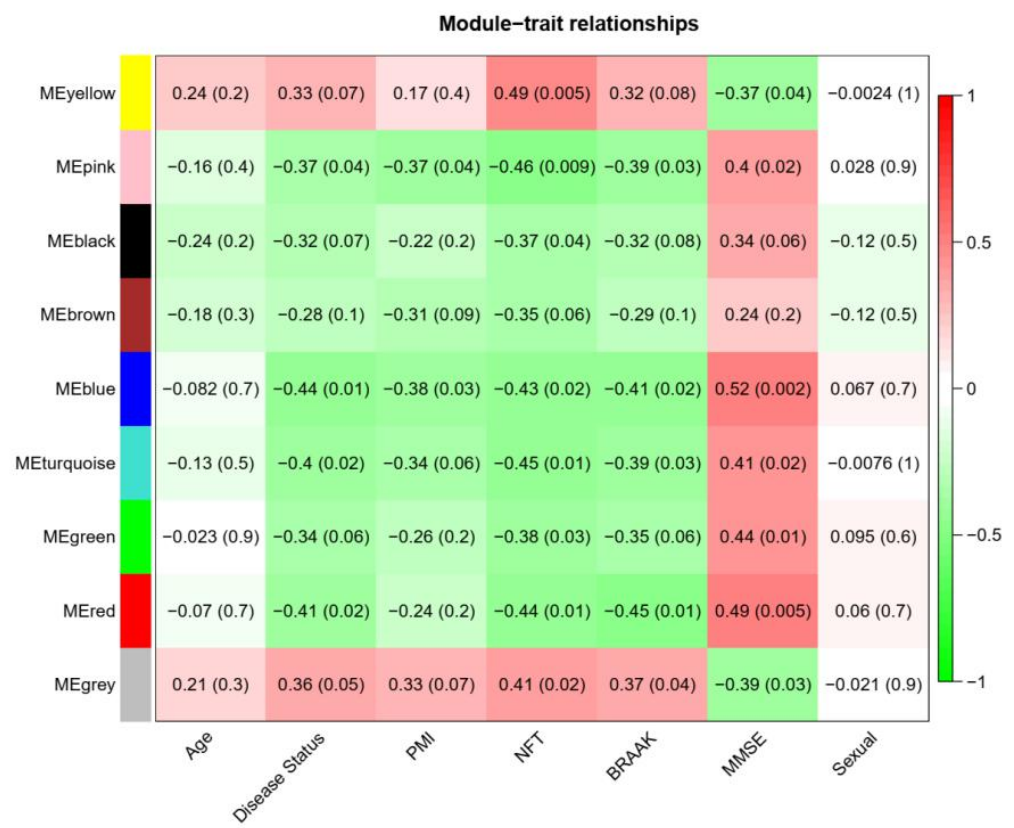

(D)

FIGURE 4| Modules related to the clinical traits of alzheimer disease. (A) Cluster dendrogram of genes: Heatmap of co-expressed genes. Different colors shown on $\mathrm{X}$ and $\mathrm{Y}$ axes denote different modules; (B) Heat map of co-expressed genes. Different colors on $\mathrm{X}$ and $\mathrm{Y}$ axes represent different modules. The depth of yellow color indicates the degree of connection between modules; (C) Dendrogram of ME obtained through WGCNA; (D) Correlation between modules and traits. Red and blue represents high adjacency and low adjacency, respectively. PMI, postmortem interval; NFT, neurofibrillary tangle; MMSE, mini-mental state examination. 


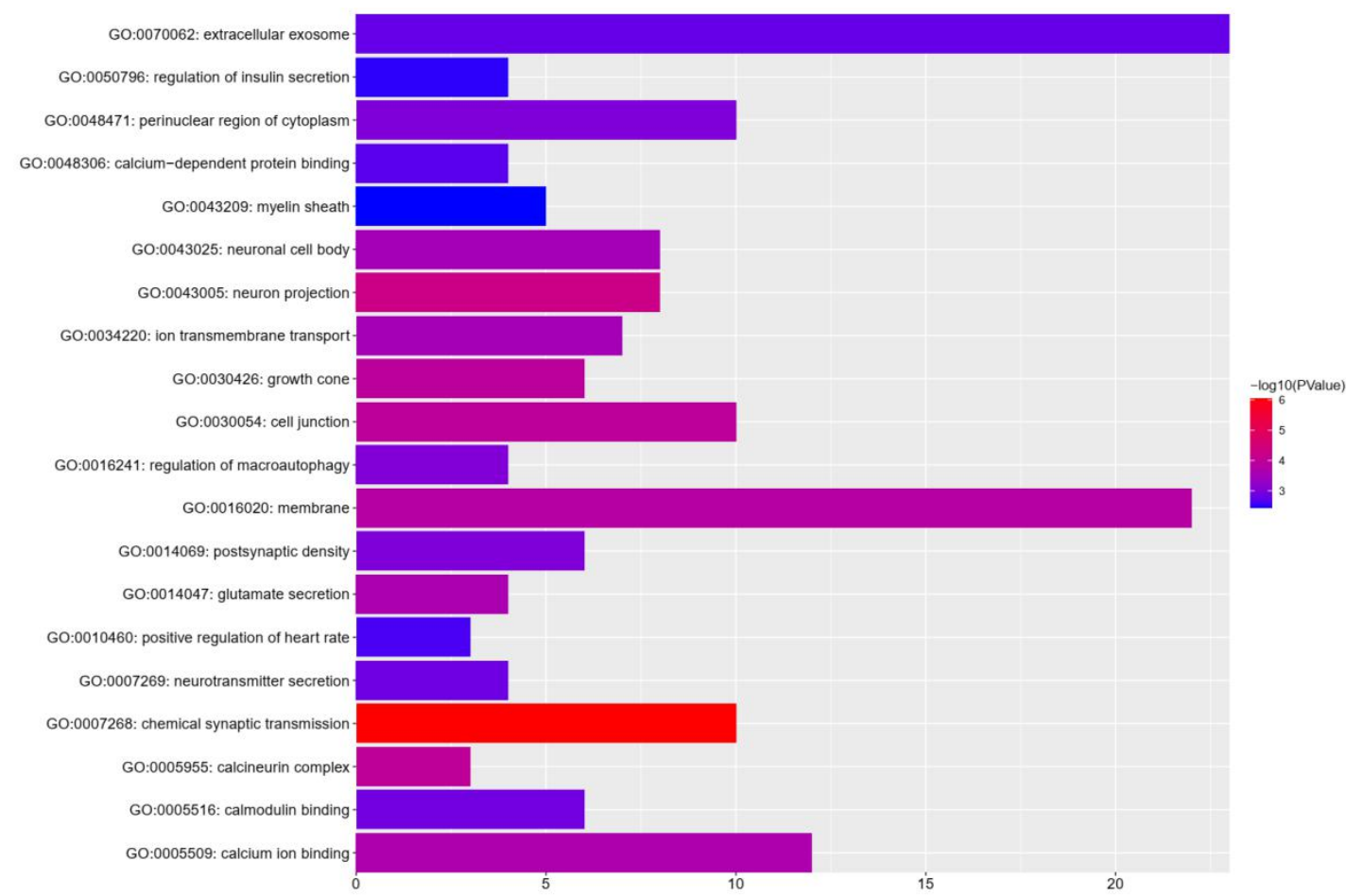

(A)

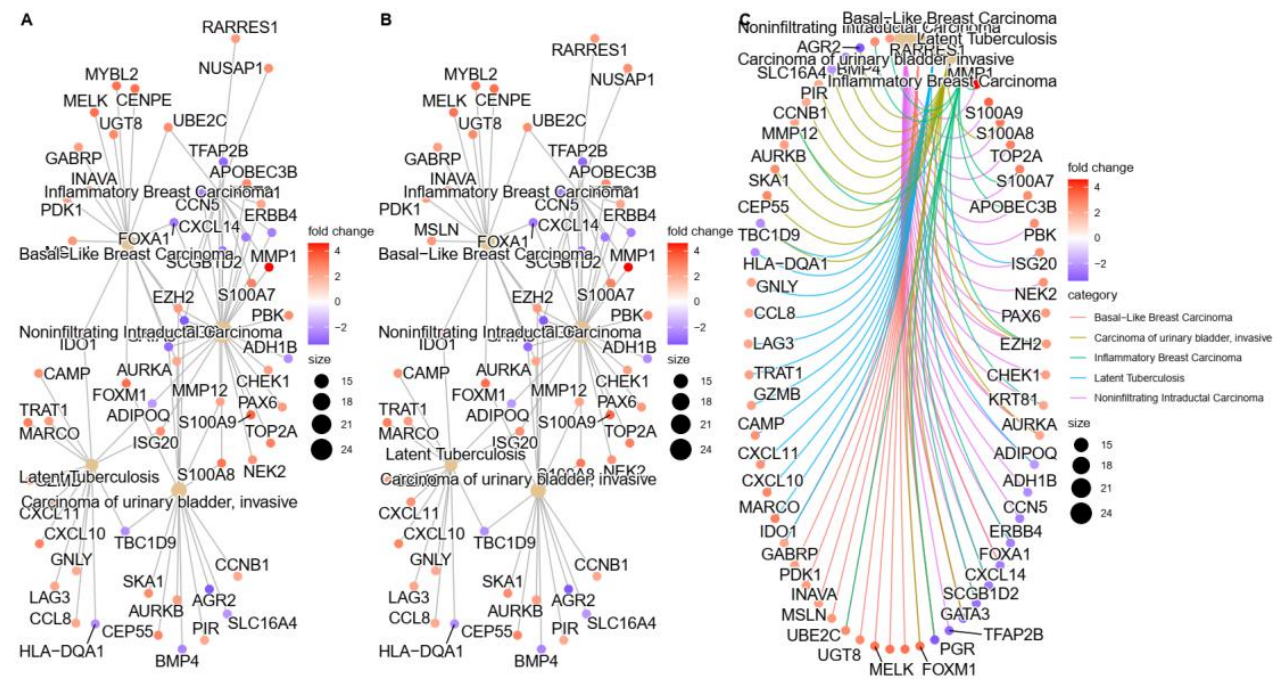




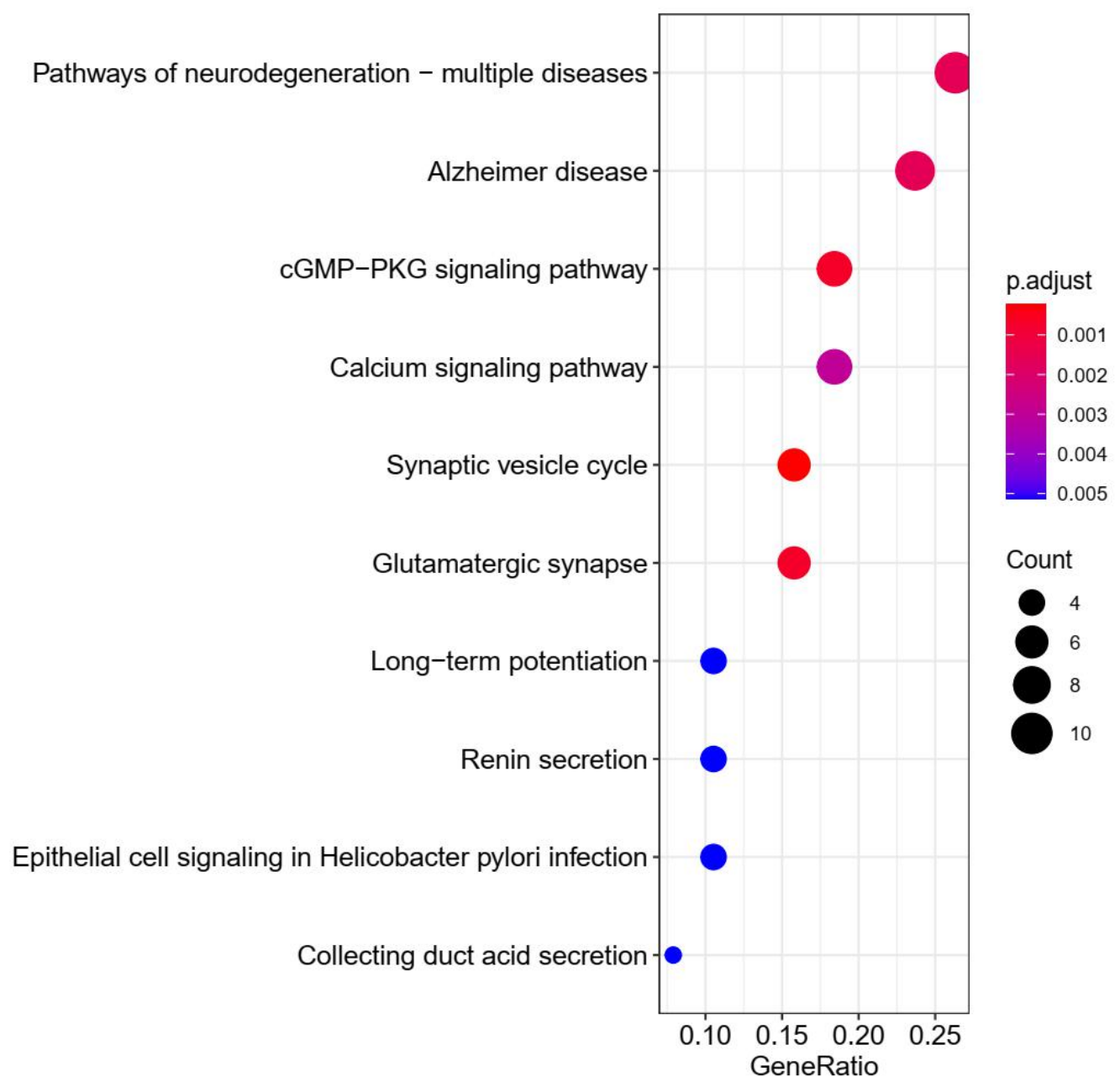

(C)
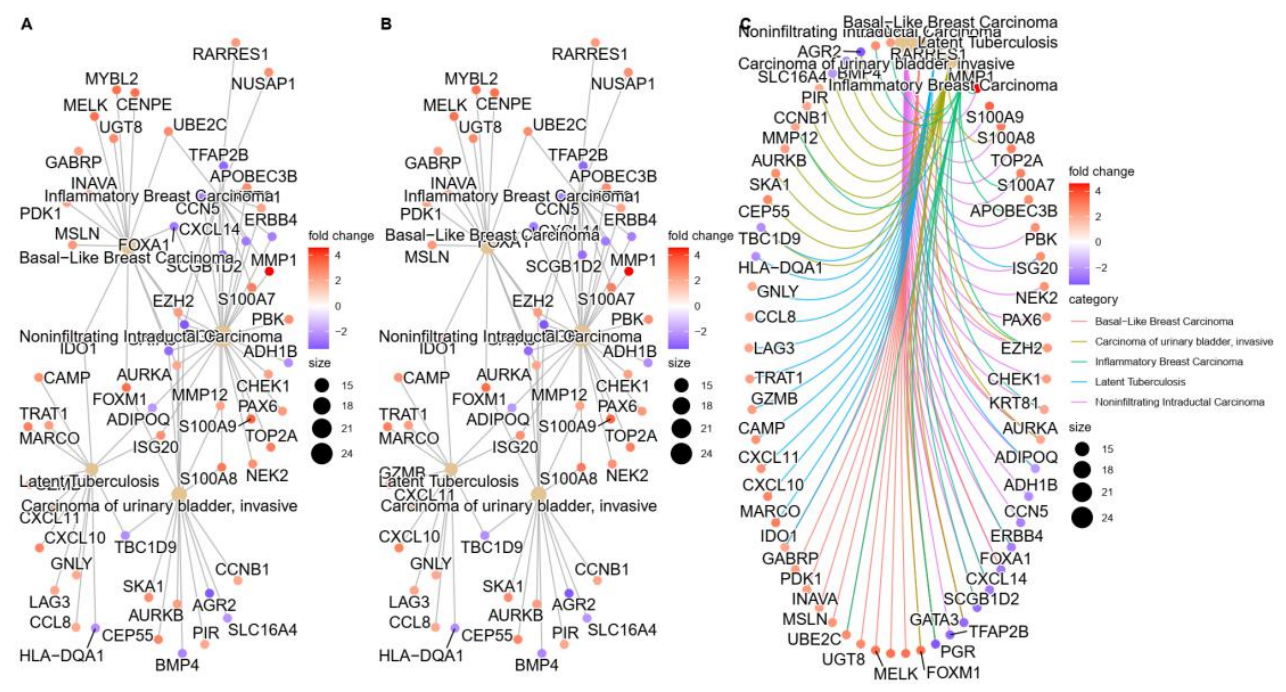


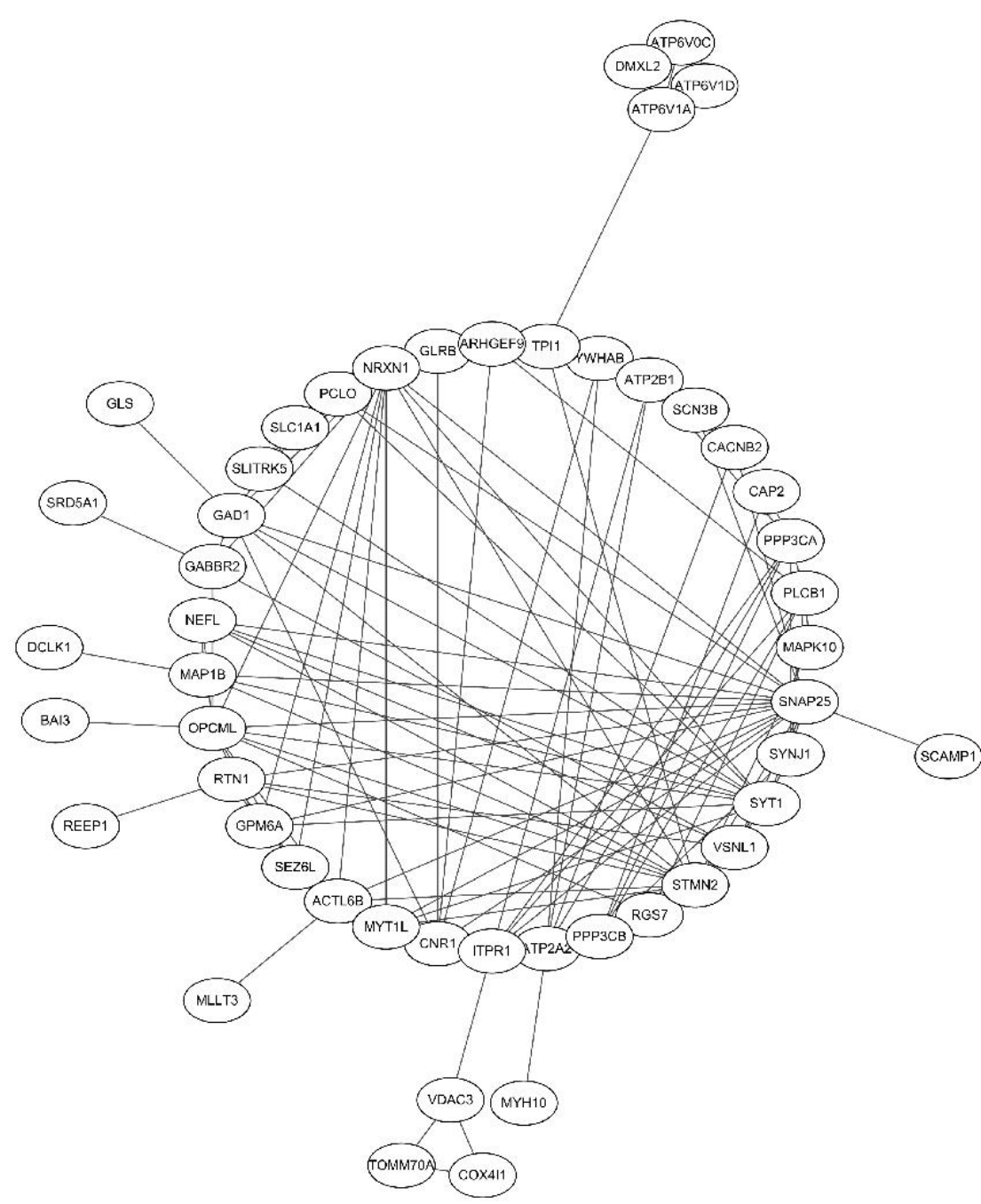

(E)

FIGURE $5 \mid$ GO and KEGG analysis for genes co-expressed with mRNAs in key modules. (A, B) Bar plot and interaction network of the top 20 enriched GO terms. (C, D) Bubble chart and interaction network of the top 20 enriched KEGG pathways. (E) A PPI network. 


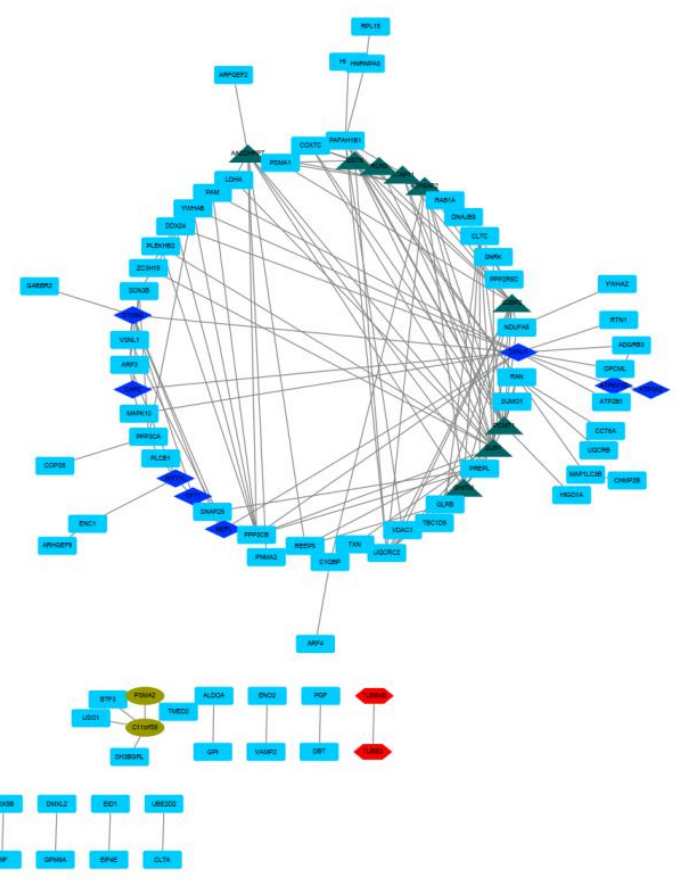

FIGURE 6| Construction of a network and hub genes. The network. Each color box represents genes within a module. The lines indicate correlation between genes.

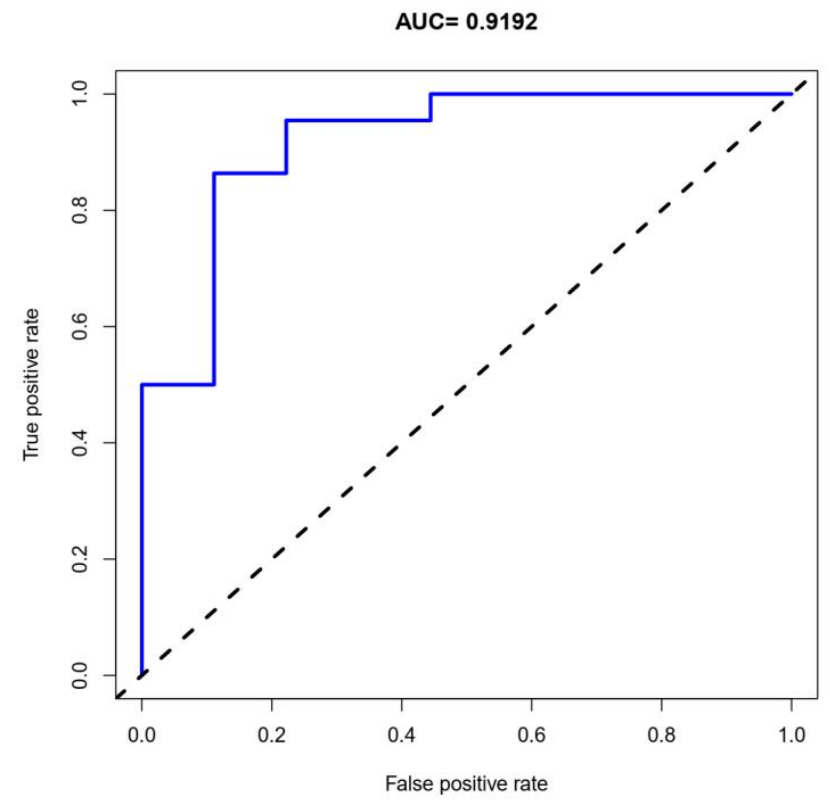

(A) 

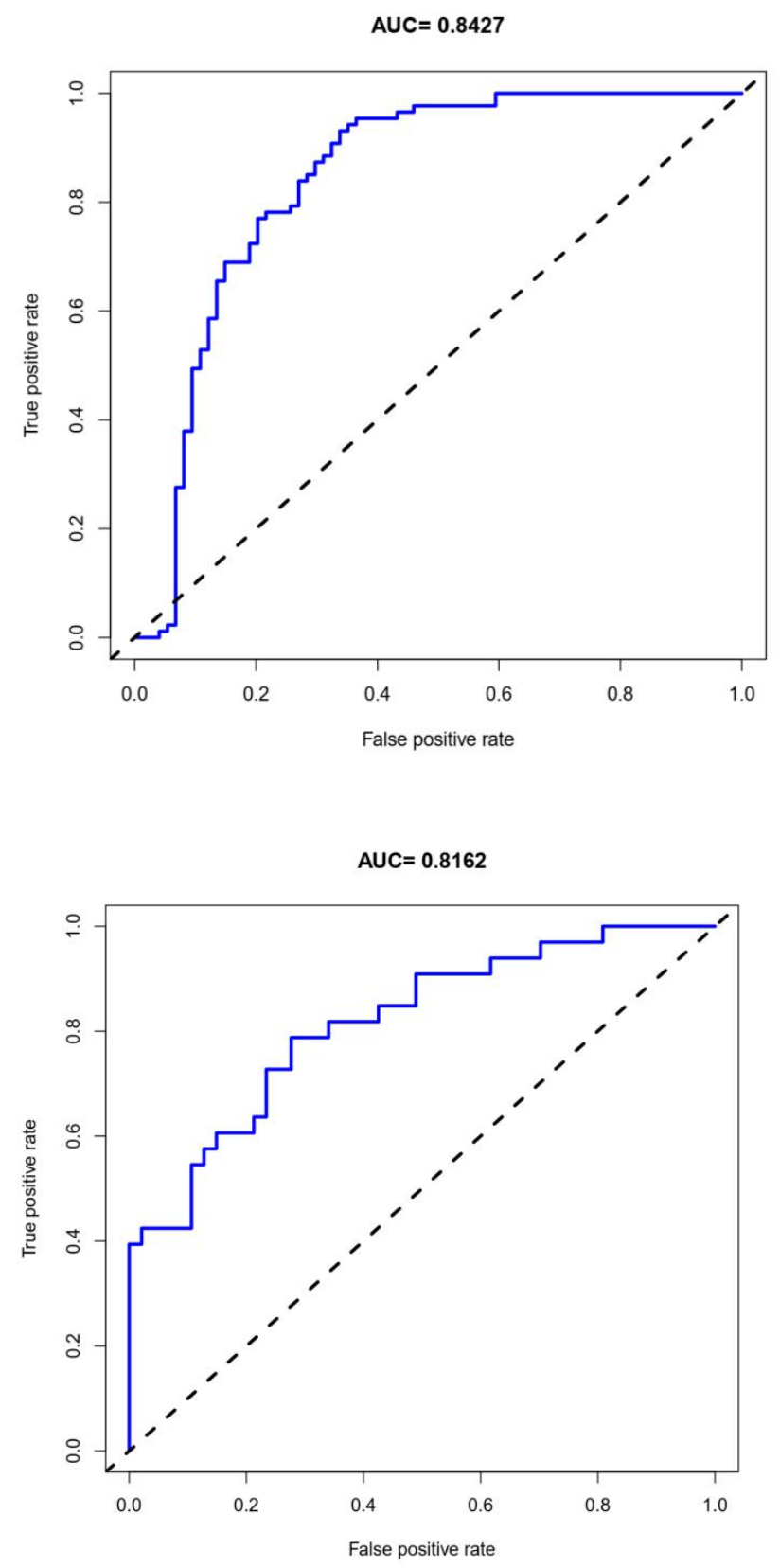

(C) 


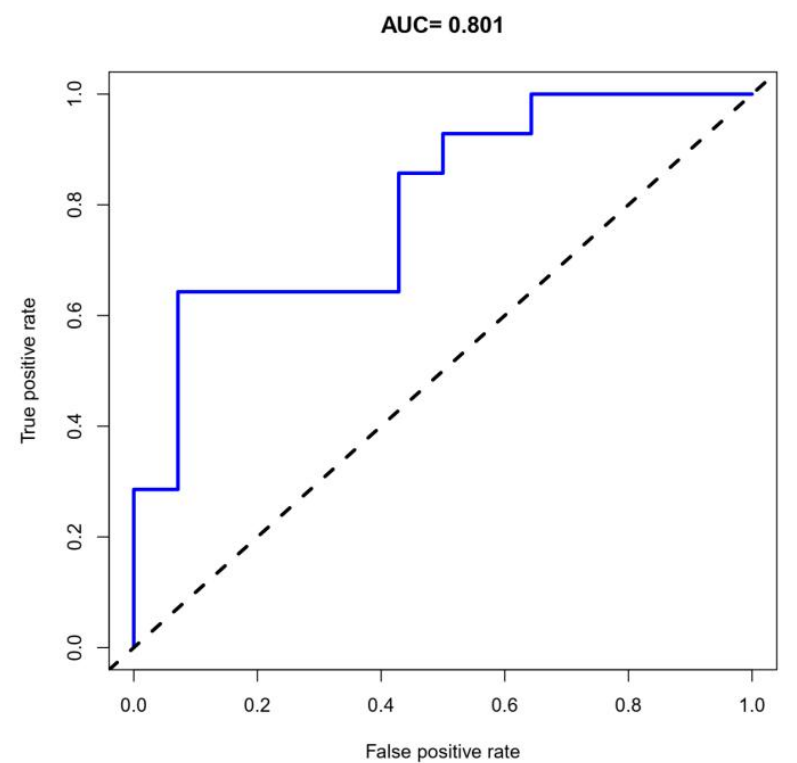

(D)

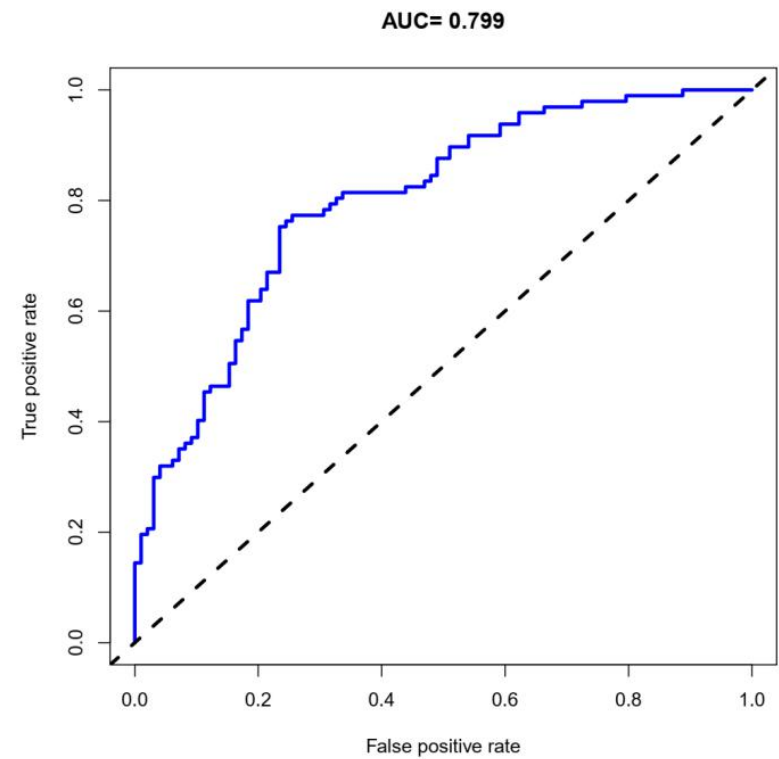

(

FIGURE 7| Receiver Operator Characteristics curves for samples in each dataset: (A) GSE1297 $(\mathrm{AUC}=0.9192) ; \quad$ (B) $\mathrm{GSE} 5281 \quad(\mathrm{AUC}=0.8427) ; \quad$ (C) GSE36980 $\quad$ (AUC=0.8162); (D)GSE4226(AUC=0.801); (E) GSE132903 (AUC=0.799). 


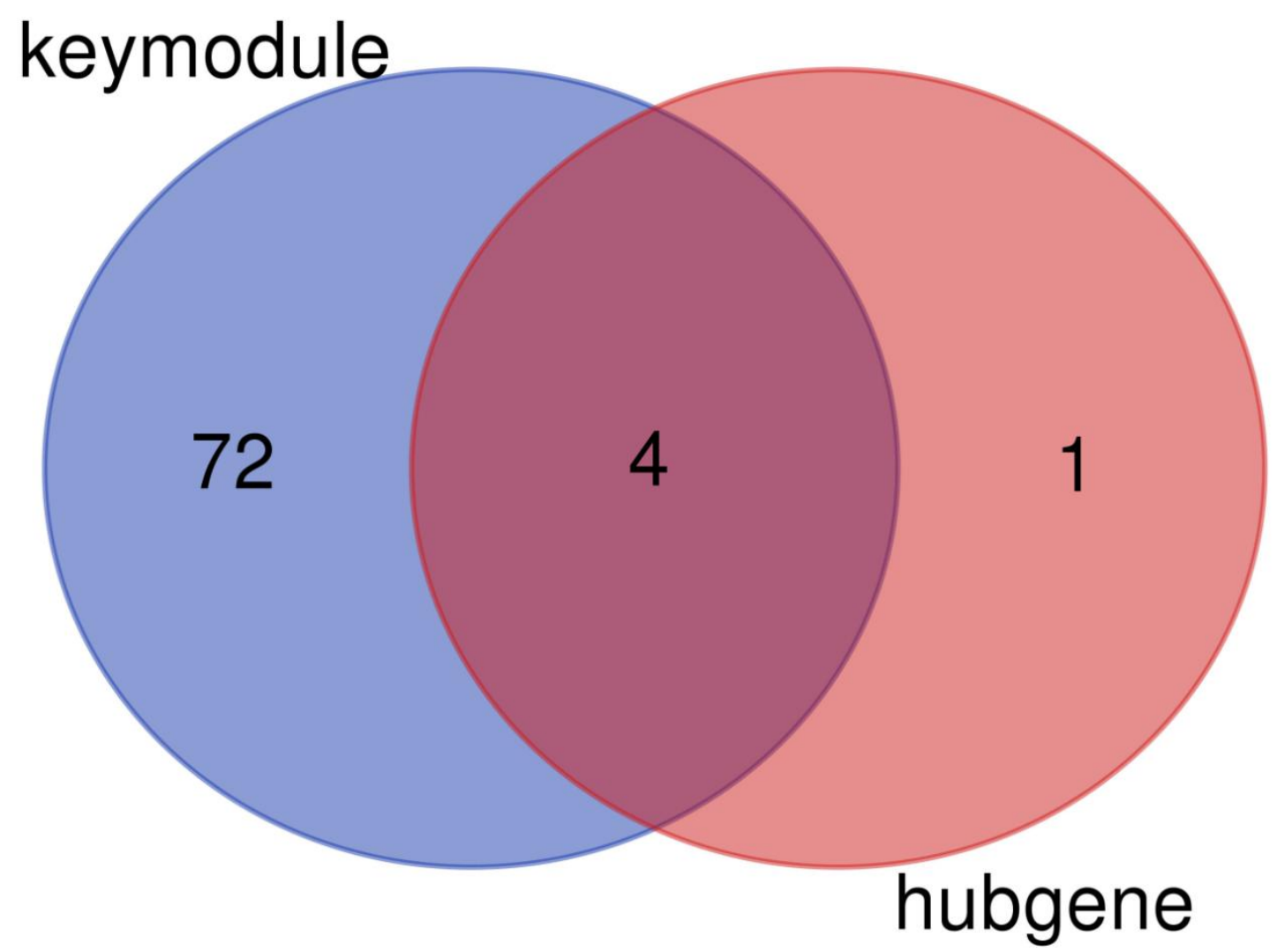

FIGURE $8 \mid$ Venn diagram. The purple Venn diagram represents genes in the key module (blue module). The red Venn diagram represents hub genes.

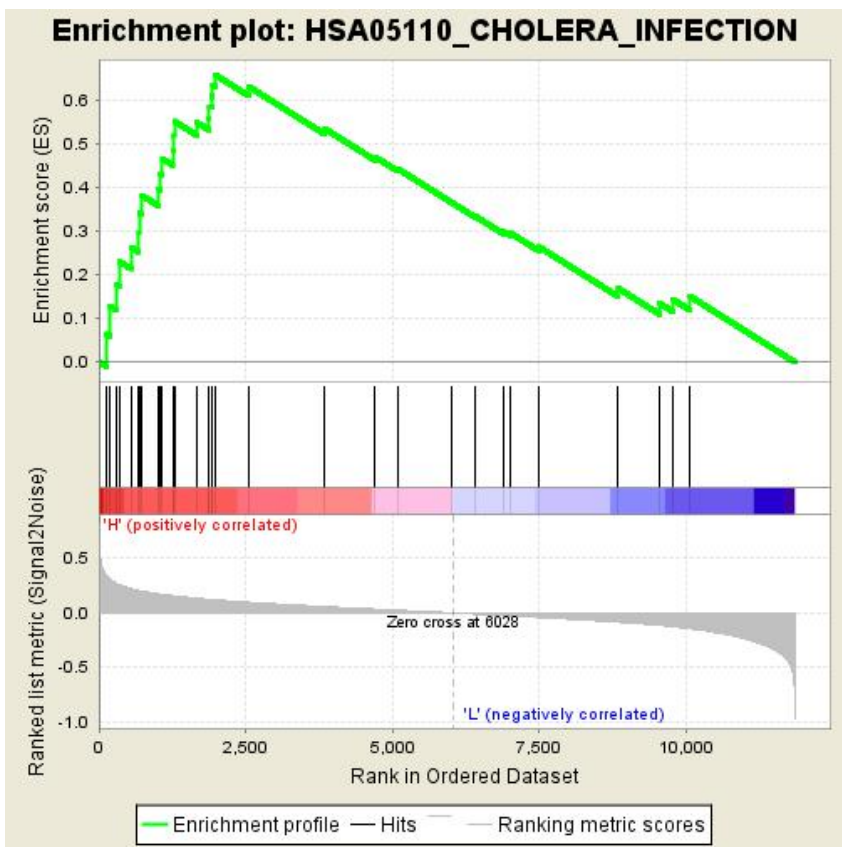

(A) 
Enrichment plot: HSA00251_GLUTAMATE_METABOLISM

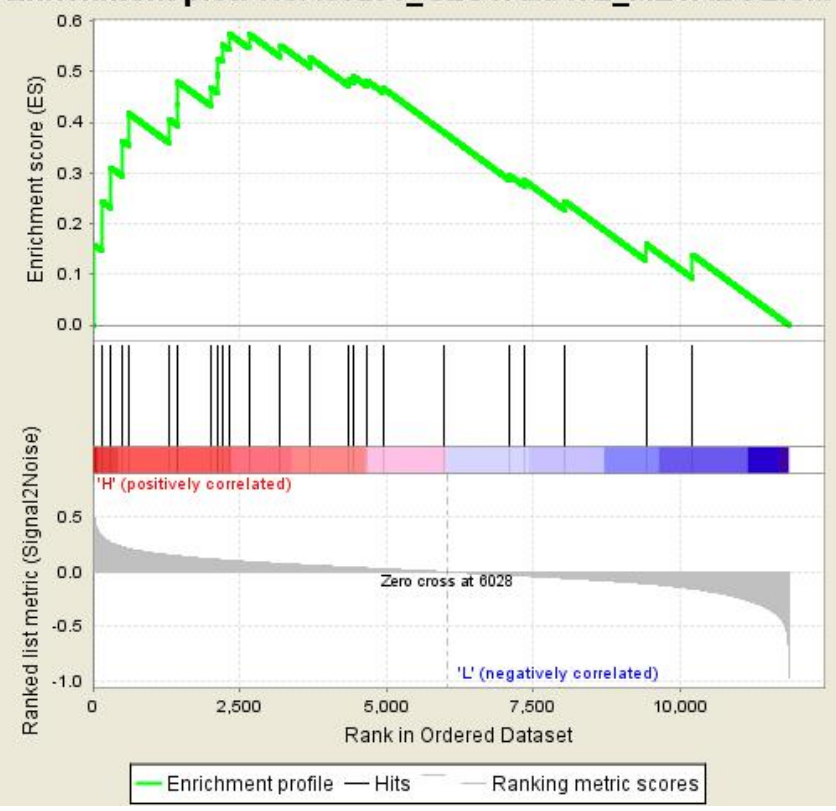

(B)

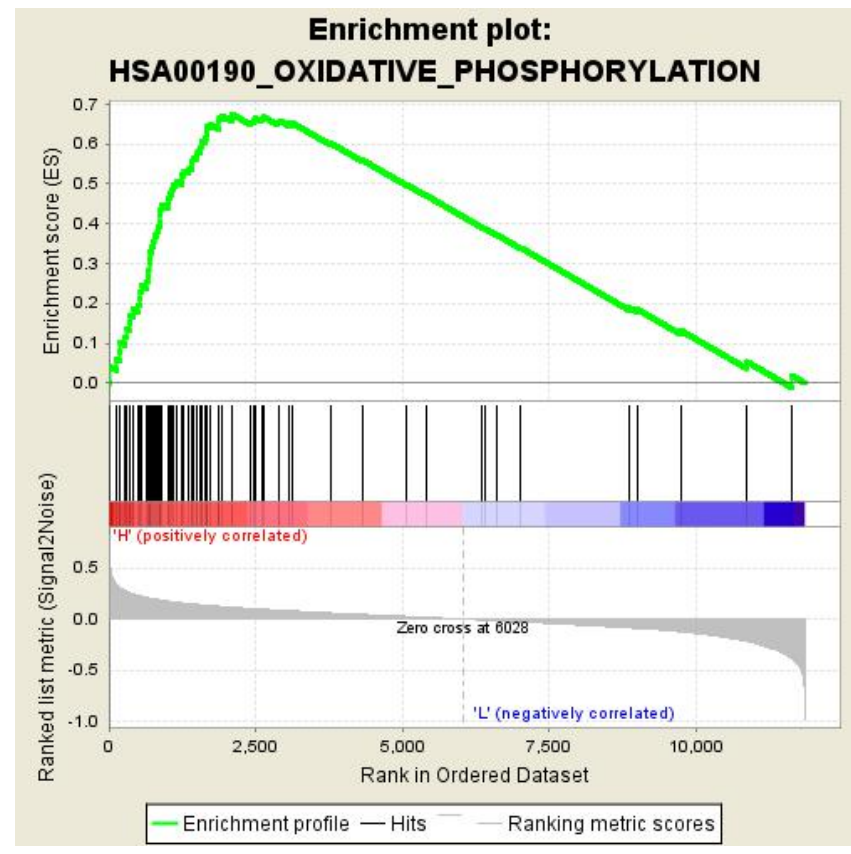

(C) 


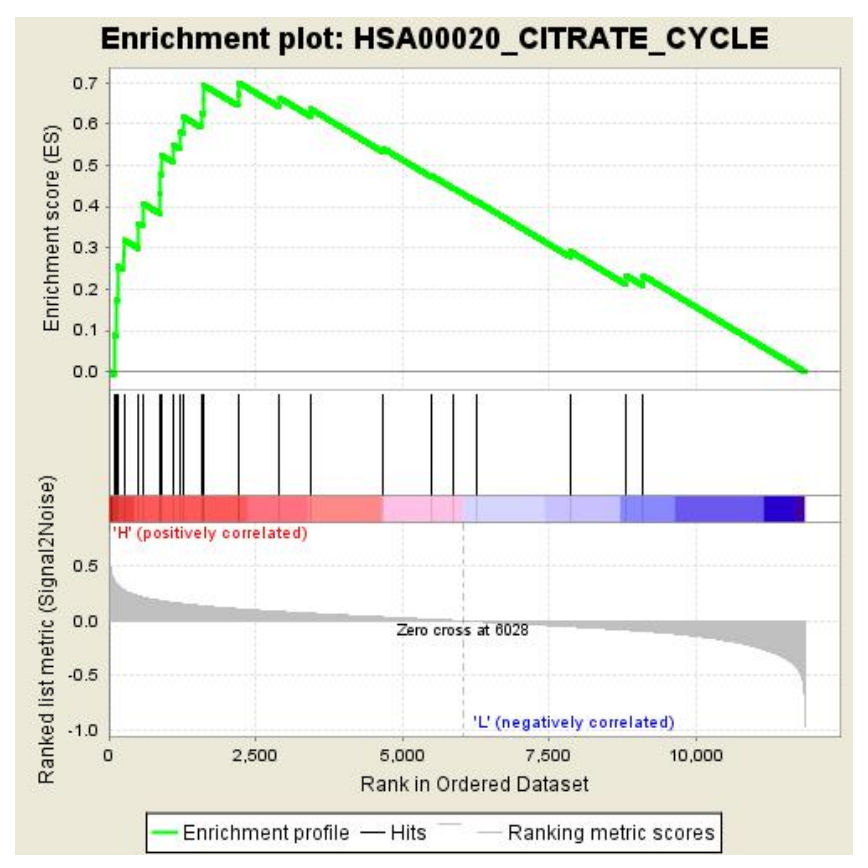

(D)

Enrichment plot:

HSA05120_EPITHELIAL_CELL_SIGNALING_IN_HELICOB ACTER_PYLORI_INFECTION

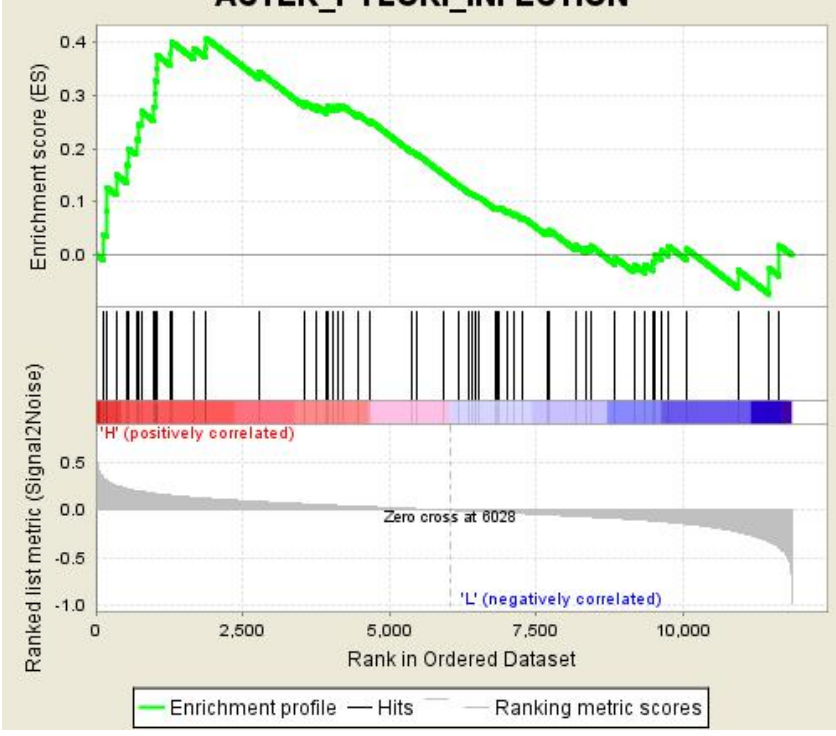

(E)

FIGURE 9| Results of Gene set enrichment analysis for the four genes (GSEA, www.broadinstitute.org/gsea/,KEGG pathways). (A) Cholera infection; (B) Glutamate metabolism; (C) Oxidative phosphorylation; (D) Citrate cycle; (E) Epithelial cell signaling in Helicobacter pylori infection. 


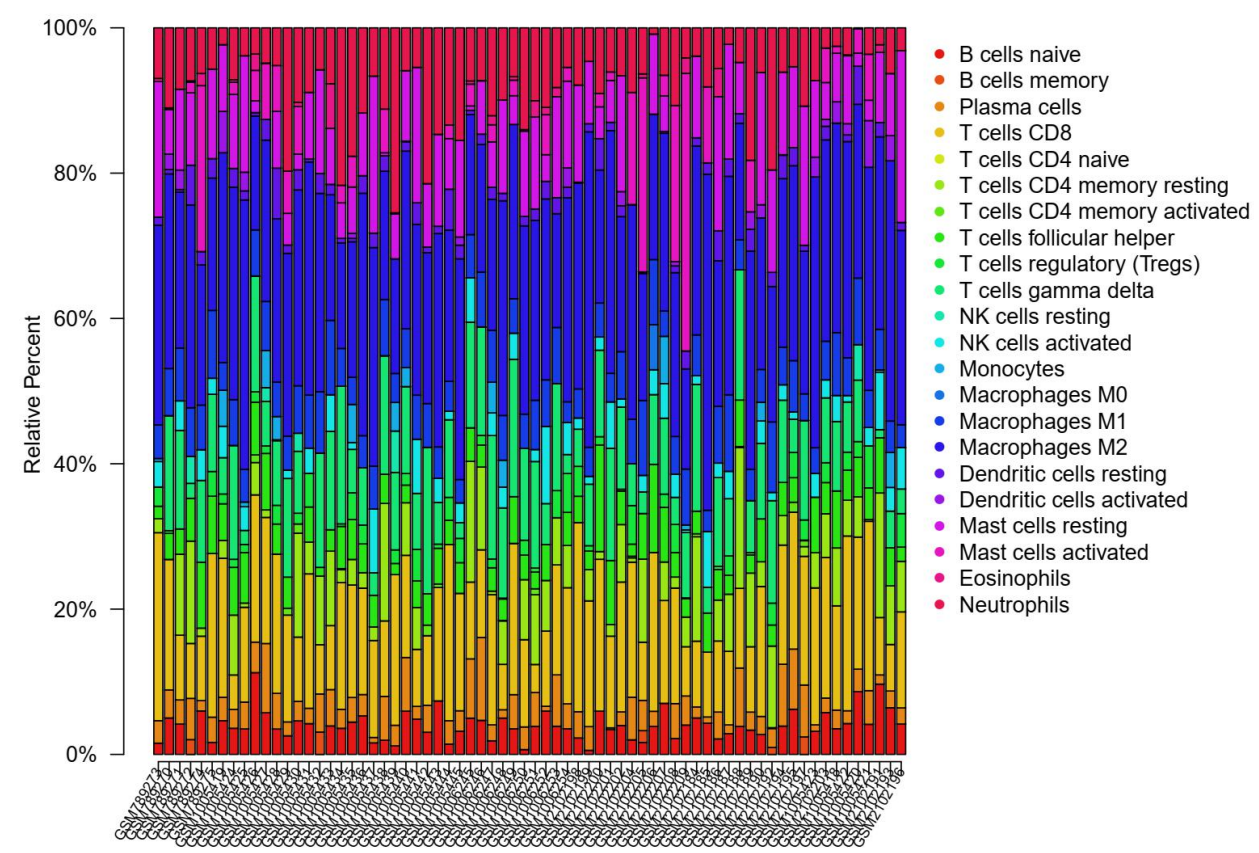

(A)

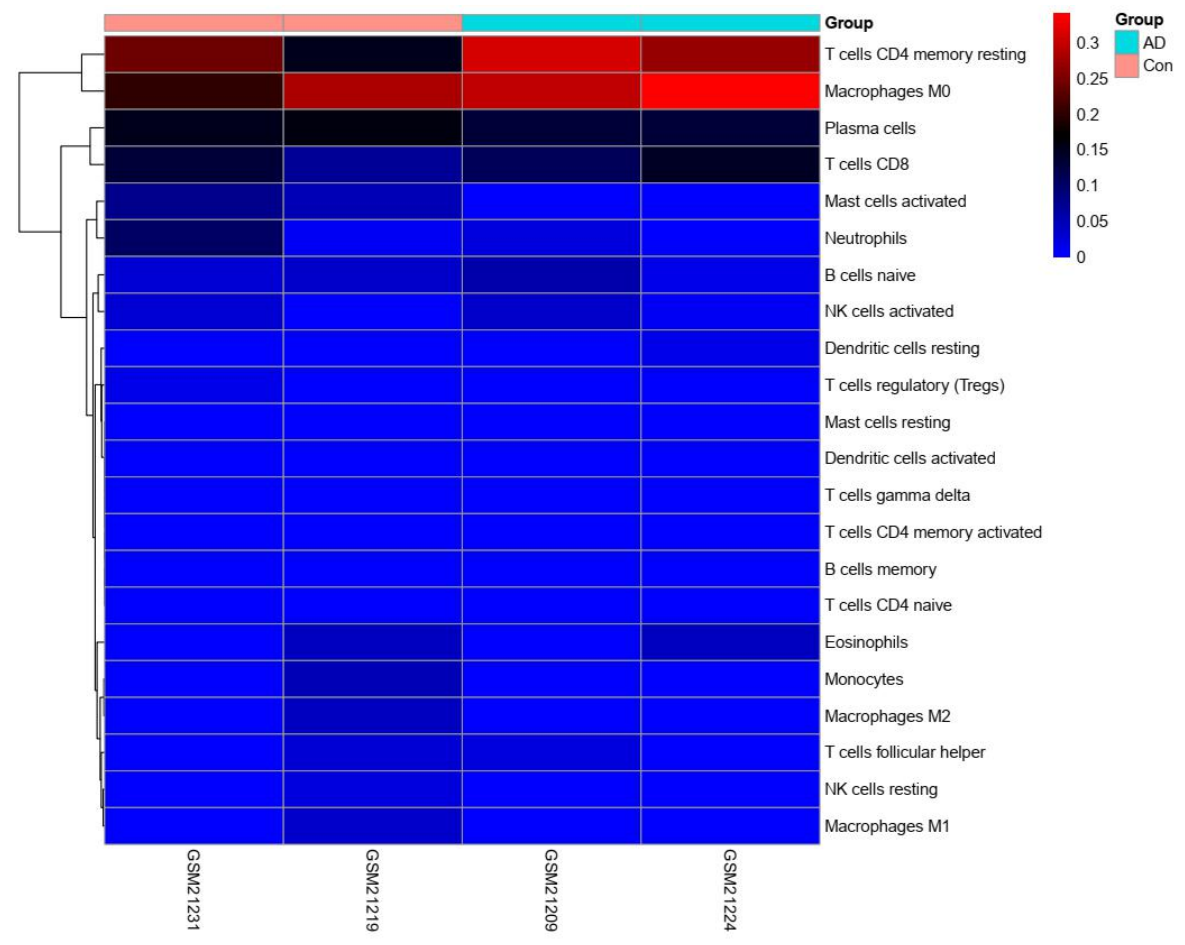

(B) 


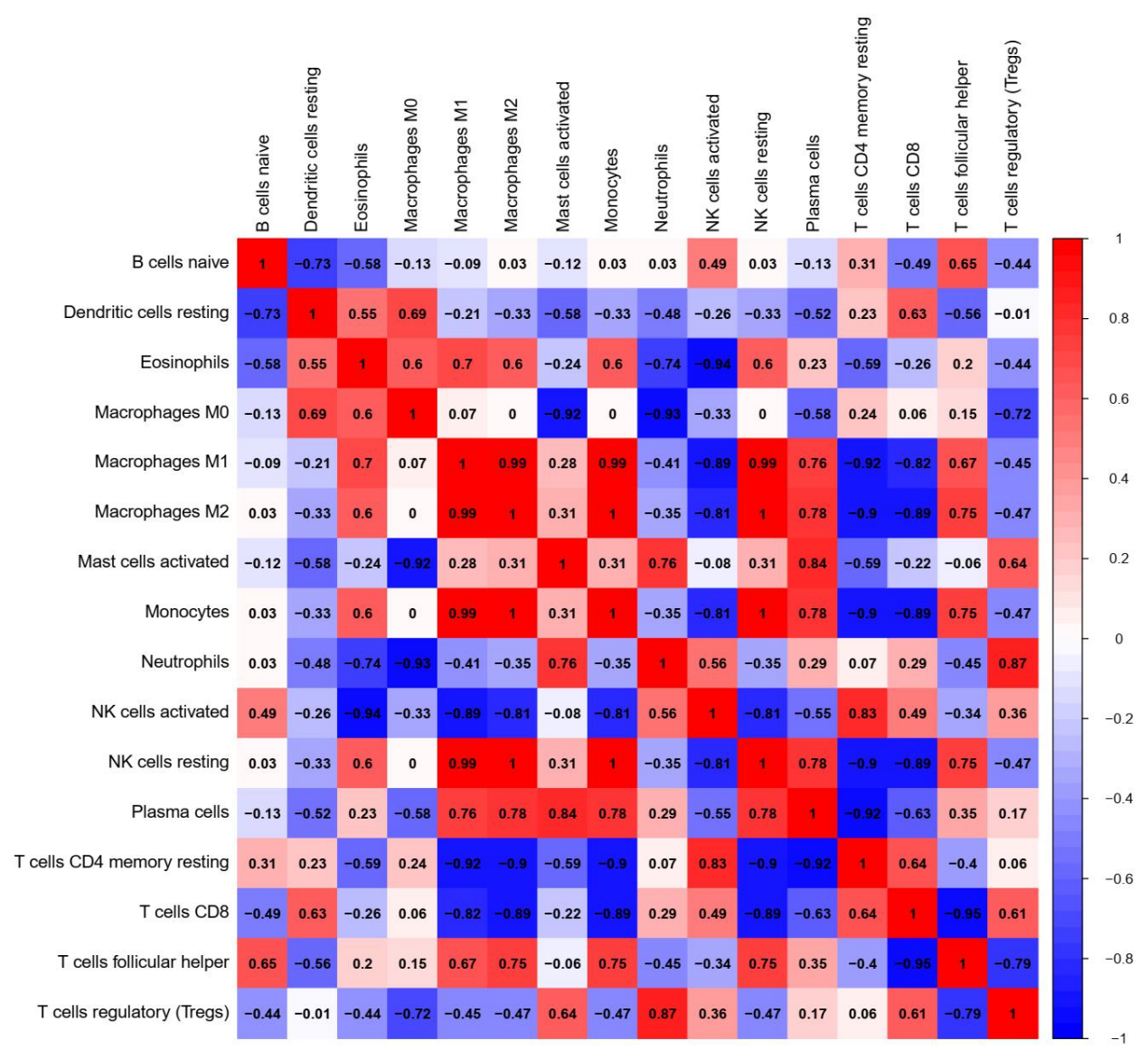

FIGURE 10| Immune infiltration landscape for alzheimer disease and normal controls. (A) The relative percentage of 22 types of immune cells. (B) A heat map of 22 types of immune cells. (C) Distribution and visualization of immune cell infiltration. Correlation matrix showing the composition of all 22 immune cell subtypes. Horizontal and vertical axes represent immune cell subtypes. High, low, and unchanged immune cell subtype compositions are shown in red, blue, and white, respectively. 